Ann. Scient. Éc. Norm. Sup.,

$4^{\text {e }}$ série, t. 39, 2006, p. 841 à 869 .

\title{
PROPER GROUPOIDS AND MOMENTUM MAPS: LINEARIZATION, AFFINITY, AND CONVEXITY
}

\author{
BY NGUYEN TIEN ZUNG
}

Dedicated to Alan Weinstein on the occasion of his 60th birthday

\begin{abstract}
We show that proper Lie groupoids are locally linearizable. As a consequence, the orbit space of a proper Lie groupoid is a smooth orbispace (a Hausdorff space which locally looks like the quotient of a vector space by a linear compact Lie group action). In the case of proper (quasi-)symplectic groupoids, the orbit space admits a natural integral affine structure, which makes it into an affine orbifold with locally convex polyhedral boundary, and the local structure near each boundary point is isomorphic to that of a Weyl chamber of a compact Lie group. We then apply these results to the study of momentum maps of Hamiltonian actions of proper (quasi-)symplectic groupoids, and show that these momentum maps preserve natural transverse affine structures with local convexity properties. Many convexity theorems in the literature can be recovered from this last statement and some elementary results about affine maps.
\end{abstract}

(c) 2006 Elsevier Masson SAS

RÉSUMÉ. - Nous montrons que les groupoïdes de Lie propres sont localement linéarisables. En particulier, l'espace des orbites d'un groupoïde de Lie propre est localement isomorphe au quotient d'un espace vectoriel par une action linéaire d'un groupe de Lie compact. Dans le cas des groupoïdes (quasi-)sympletiques propres, l'espace des orbites est une orbifold qui admet une structure affine naturelle avec des propriétés de convexité locale. Nous appliquons ces résultats à l'étude des actions hamiltoniennes de groupoïdes (quasi-)symplectiques propres, et montrons que les applications moment de telles actions ont un caractère affine et des propriétés de convexité. Nous retrouvons plusieurs théorèmes de convexité bien connus dans ce contexte.

(c) 2006 Elsevier Masson SAS

\section{Introduction}

This paper consists of two parts. The first part is about the linearization problem for Lie groupoids and (quasi-)symplectic groupoids. The main result of this part is the local linearization theorem (Theorem 2.3), which states that any proper Lie groupoid with a fixed point is locally linearizable, i.e. locally isomorphic to the action groupoid of a linear action of a compact Lie group on a vector space. A consequence of this local linearization theorem is the slice theorem (Theorem 2.4), which linearizes a proper Lie groupoid in a neighborhood of an orbit under two additional conditions: that the groupoid is source-locally trivial, and the orbit in question is a manifold of finite type. This slice theorem was obtained by Weinstein [31] modulo Theorem 2.3, and is a generalization of the classical Koszul-Palais' slice theorem for proper Lie group actions $[18,26]$ to the case of Lie groupoids. Another immediate consequence of the local linearization 
theorem is that the characteristic foliation on the base space of a proper Lie groupoid is an orbitlike foliation in the sense of Molino [24] with closed orbits, and the corresponding orbit space (= space of orbits) is a smooth orbispace in the sense that it is a Hausdorff space which is locally smoothly isomorphic to the quotient of a vector space by a linear action of a compact Lie group. In the case of symplectic groupoids, Theorem 2.3 together with some standard arguments imply that a slice of a proper symplectic groupoid is locally isomorphic to a standard symplectic groupoid $T^{*} G \rightrightarrows \mathfrak{g}^{*}$, where $G$ is a compact Lie group and $\mathfrak{g}$ its Lie algebra (Theorem 2.5), and the orbit space is a manifold with boundary which looks locally like a Weyl chamber (under the additional condition that the isotropy groups are "coad-connected", i.e. their coadjoint orbits are connected; if this condition is not satisfied then the orbit space is an orbifold). A similar result (Corollary 2.6) holds for quasi-symplectic groupoids in the sense of Xu [32] (a.k.a. twisted presymplectic groupoids [3]).

The second part of this paper is about the convexity properties of momentum maps in symplectic geometry. We will consider momentum maps in the context of Hamiltonian spaces of quasi-symplectic groupoids [32], an approach which unifies the classical theory of equivariant momentum maps for Hamiltonian group actions, Lu's momentum map theory for actions of Poisson-Lie groups [19], and also Alekseev-Malkin-Meinrenken's theory of group-valued momentum maps [1]. Actually, what we want to emphasize in this paper is not the convexity, but the affinity of momentum maps. More precisely, we will show that if $\Gamma \rightrightarrows P$ is a proper quasi-symplectic groupoid, then $P$ together with its characteristic (singular) foliation admits a natural transverse integral flat affine structure (which projects to an affine structure on the orbit space), and any Hamiltonian $\Gamma$-space also admits a natural transverse affine structure to a singular "coisotropic" foliation associated to the action of $\Gamma$; the momentum map sends the leaves of this foliation to the orbits of $P$, and is transversally affine, i.e. it preserves the transverse affine structure. One then recovers various known momentum map convexity theorems from this affine property, local convexity and some elementary results concerning affine maps between locally convex affine spaces.

\section{Proper groupoids}

\subsection{Linearization of proper groupoids}

Let us start by formulating the linearization problem. Consider a Lie groupoid $\mathcal{G} \rightrightarrows M$. We will always denote the source map and the target map by $s$ and $t$ respectively. Consider an orbit $\mathcal{O}$ of $\mathcal{G}$ on $M$. Then the restriction $\mathcal{G}_{\mathcal{O}}:=\{p \in \mathcal{G} \mid s(p), t(p) \in \mathcal{O}\}$ of $\mathcal{G}$ to $\mathcal{O}$ is a transitive Lie groupoid over $\mathcal{O}$, and the structure of $\mathcal{G}$ induces a linear action of $\mathcal{G}_{\mathcal{O}}$ on the normal vector bundle $N_{\mathcal{O}}$ of $\mathcal{O}$ in $M$. (This action may be defined as follows: let $g \in \mathcal{G}_{\mathcal{O}}$ and $x \in N_{\mathcal{O}}$ such that $s(g)$ coincides with the projection of $x$ to $\mathcal{O}$. Let $\alpha$ be a parametrized curve in $M$ such that $\alpha(0)=s(g)$ and $\left.\frac{\mathrm{d}}{\mathrm{d} \varepsilon} \alpha(\varepsilon)\right|_{\varepsilon=0}$ projects to $x$. Let $\gamma$ be a parametrized curve in $\Gamma$ such that $\gamma(0)=g$ and $s(\gamma)=\alpha$. Then the projection $y$ of $\left.\frac{\mathrm{d}}{\mathrm{d} \varepsilon} t(\gamma(\varepsilon))\right|_{\varepsilon=0}$ to $N_{\mathcal{O}}$ does not depend on the choice of $\alpha$ and $\gamma$, and by definition $g . x=y$.) The corresponding semi-direct product $\mathcal{G}_{\mathcal{O}} \ltimes N_{\mathcal{O}}$ is the linear model for $\mathcal{G}$ in the neighborhood of $\mathcal{O}$. The question is: do there exist a neighborhood $U$ of $\mathcal{O}$ in $M$ and a neighborhood $V$ of the zero section in $N_{\mathcal{O}}$ such that the restriction $\mathcal{G}_{U}=\{p \in G \mid s(p), t(p) \in U\}$ of the groupoid $\mathcal{G}$ to $U$ is isomorphic to $\left(\mathcal{G}_{\mathcal{O}} \ltimes N_{\mathcal{O}}\right)_{V}$ ?

This linearization problem is a generalization of the problem of linearization of Lie group actions. A special case is when $\mathcal{G}=G \ltimes M$ is the action groupoid of an action of a Lie group $G$ on a manifold $M$ with a fixed point $m \in M$. Then the linearization of $\mathcal{G}$ near $m$ is similar though somewhat weaker than the linearization of the action of $G$ on $M$ near $m$ : if the action of $G$ is

$4^{e}$ SÉRIE - TOME $39-2006-\mathrm{N}^{\circ} 5$ 
linearized then the corresponding action Lie groupoid is also linearized, and conversely if the action groupoid $G \ltimes M$ is linearized then it means that the action of $G$ is "orbitally linearized", i.e. its orbits are the same as that of a linear action, though the action of $G$ itself may still be nonlinear.

The classical theorems of Bochner [2], Koszul [18] and Palais [26] say that, under a compactness or properness condition, smooth Lie group actions can be linearized (near a fixed point or an orbit). On the other hand, it is easy to construct non-proper actions (for $G=\mathbb{R}$ for example) which cannot be linearized, not even orbitally. For these reasons, in this paper we will restrict our attention to proper groupoids.

Definition 2.1 [31]. - A Lie groupoid $\mathcal{G} \rightrightarrows M$ is called proper if $\mathcal{G}$ is Hausdorff and the map $(s, t): \mathcal{G} \rightarrow M \times M$ is a proper topological map, i.e. the preimage of a compact set is compact.

Remark. - By convention, the base space (= space of objects) $M$ of a Lie groupoid $\mathcal{G} \rightrightarrows M$ is always Hausdorff, but the space of arrows $\mathcal{G}$ is a not-necessarily-Hausdorff manifold. However, all groupoids in this paper are assumed to be Hausdorff even when not mentioned explicitly.

For example, the action groupoid $G \ltimes M$ of a smooth action of a Lie group $G$ on a manifold $M$ is a proper Lie groupoid if and only if the action of $G$ on $M$ is proper, by definition.

The above properness condition has some immediate topological consequences, which we put together into a proposition:

PROPOSITION 2.2 [23,31]. - Let $\mathcal{G} \rightrightarrows M$ be a proper Lie groupoid. Then we have:

(i) The isotropy group $G_{m}=\{p \in \mathcal{G} \mid s(p)=t(p)=m\}$ of any point $m \in M$ is a compact Lie group.

(ii) Each orbit $\mathcal{O}$ of $\mathcal{G}$ on $M$ is a closed submanifold of $M$.

(iii) The orbit space $M / \mathcal{G}$ together with the induced topology is a Hausdorff space.

(iv) If $\mathcal{H}$ is a Hausdorff Lie groupoid which is Morita-equivalent to $\mathcal{G}$ then $\mathcal{H}$ is also proper.

(v) If $N$ is a submanifold of $M$ which intersects an orbit $\mathcal{O}$ transversally at a point $m \in M$, and $B$ is a sufficiently small open neighborhood of $m$ in $N$, then the restriction $\mathcal{G}_{B}=s^{-1}(B) \cap t^{-1}(B)$ is a proper Lie groupoid which has $m$ as a fixed point.

Proof. - Points (i) and (v) follow directly from the definition. A sketchy proof of point (iv) can be found in Chapter 5 of [23]. Point (ii), which was proved in [31], is a corollary of point (iii). Let us give here a proof of point (iii): Let $x, y \in M$ such that their orbits are different: $\mathcal{O}(x) \cap \mathcal{O}(y)=\emptyset$, or equivalently, $s^{-1}(y) \cap t^{-1}(x)=\emptyset$. Denote by $D_{1}^{z} \supset D_{2}^{z} \supset \cdots \ni z$ a series of compact neighborhoods (i.e. compact sets which contain open neighborhoods) of $z$ in $M$, where $z=x$ or $y$, such that $\bigcap_{n \in \mathbb{N}} D_{n}^{z}=\{z\}$. We have $\bigcap_{n \in \mathbb{N}} t^{-1}\left(D_{n}^{x}\right) \cap s^{-1}\left(D_{n}^{y}\right)=$ $t^{-1}(x) \cap s^{-1}(y)=\emptyset$. Since $\Gamma$ is proper, the sets $t^{-1}\left(D_{n}^{x}\right) \cap s^{-1}\left(D_{n}^{y}\right)$ are compact. It follows that there is $n \in \mathbb{N}$ such that $t^{-1}\left(D_{n}^{x}\right) \cap s^{-1}\left(D_{n}^{y}\right)=\emptyset$, or equivalently, $\mathcal{O}\left(D_{n}^{x}\right) \cap \mathcal{O}\left(D_{n}^{y}\right)=\emptyset$, where $\mathcal{O}\left(D_{n}^{x}\right)$ is the union of orbits through $D_{n}^{x}$. But the orbit space of $\mathcal{O}\left(D_{n}^{x}\right)\left(\right.$ resp. $\left.\mathcal{O}\left(D_{n}^{y}\right)\right)$ is a (compact) neighborhood of $x$ (resp. $y$ ) in the orbit space of $M$. Thus the orbit space of $M$ is Hausdorff.

The groupoid $\mathcal{G}_{B}$ in point (v) of the above proposition is called a slice of $\mathcal{G}$ at $m$. This notion makes sense even when $\mathcal{G}$ is not proper. Two Lie groupoids $\Gamma_{1} \rightrightarrows B_{1}$ and $\Gamma_{2} \rightrightarrows B_{2}$ with fixed points $m_{1} \in B_{1}$ and $m_{2} \in B_{2}$ are called locally isomorphic (near $m_{1}$ and $m_{2}$ ) if there are open neighborhoods $U_{1}$ of $m_{1}$ in $B_{1}$ and $U_{2}$ of $m_{2}$ in $B_{2}$ such that $\left(\Gamma_{1} \rightrightarrows B_{1}\right)_{U_{1}}:=$ $\left\{p \in \Gamma_{1} \mid s(p), t(p) \in U_{1}\right\}$ is isomorphic to $\left(\Gamma_{2} \rightrightarrows B_{2}\right)_{U_{2}}$. Recall that, similarly to the case of Lie algebroids, two arbitrary slices of a groupoid at two points lying on a same orbit are locally 
isomorphic, and the local isomorphism class may be called the transverse groupoid structure to the orbit in question.

The main result of this paper is the following theorem, which was conjectured by Weinstein [29,31]:

THEOREM 2.3. - Any proper Lie groupoid $\Gamma \rightrightarrows B$ with a fixed point $m \in B$ is locally isomorphic to a linear action groupoid, namely the action groupoid of the action of the compact isotropy group $G=G_{m}$ on the tangent space $V=T_{m} B$.

Remark. - Structural maps and manifolds of Lie groupoids are usually assumed to be $C^{\infty}$-smooth, but the above theorem holds for finitely differentiable Lie groupoids as well: if $\Gamma$ is of class $C^{k}(k=1,2, \ldots, \infty)$ then it can be locally linearized by an isomorphism of class $C^{k}$. We suspect that the $C^{\omega}$ version of Theorem 2.3 is also true, though we do not have a proof of it.

Remark. - In the case when the isotropy group $G$ is semisimple, Theorem 2.3 (and its $C^{\omega}$ version) follows from the corresponding results about linearization of Lie algebroids obtained by Monnier and the author in $[33,25]$. The proof of Theorem 2.3 presented in the present paper uses an averaging method and standard Banach norm estimations, and is considerably simpler than the Kolmogorov-Nash-Moser fast convergence method used in [33,25]. We suspect that the results of $[33,25]$ might lead to a generalization of Theorem 2.3 (partial linearization of non-proper Lie groupoids). Conversely, generalizations of Theorem 2.3 and of Cranic-Fernandes' theorem about integrability of Lie algebroids [6] might lead to results about (partial) linearization of Lie algebroids and Poisson structures.

The proof of Theorem 2.3 will start in the next subsection. In the rest of this subsection, we will discuss some of its important consequences.

An immediate consequence of Theorem 2.3 is that if $\mathcal{G} \rightrightarrows M$ is a proper Lie groupoid, then the characteristic singular foliation on $M$ (by the orbits of $\mathcal{G}$ ) is an orbit-like foliation in the sense of Molino [24]. In particular, it is a singular Riemannian foliation. Moreover, the orbit space $M / \mathcal{G}$ (together with the induced topology and smooth structure from $M$ ) locally looks like the quotient of a vector space by a linear action of a compact Lie groups. (Locally, the orbit space $M / \mathcal{G}$ is the same as the orbit space of a slice $B / \mathcal{G}_{B}$.) In analogy with the fact that orbifolds are orbit spaces of étale proper groupoids [14,22], it would be natural to call the orbit space (or rather the stack) of a proper Lie groupoid a (smooth) orbispace. In the literature there are some other similar but maybe non-equivalent notions of orbispaces.

Another direct consequence of Theorem 2.3 is the following slice theorem for Lie groupoids, which was obtained by Weinstein [31, Theorem 9.1] under the hypothesis that Theorem 2.3 is true. Recall that if $\mathcal{O}$ is an orbit on $M$ of a Lie groupoid $\mathcal{G} \rightrightarrows M$, then we denote by $\mathcal{G}_{\mathcal{O}}$ the restriction of $\mathcal{G}$ to $\mathcal{O}$, and by $N_{\mathcal{O}}$ the normal bundle of $\mathcal{O}$ in $M$. There is a natural linear action of $\mathcal{G}_{\mathcal{O}}$ on $N_{\mathcal{O}}$, and we denote by $\mathcal{G}_{\mathcal{O}} \ltimes N_{\mathcal{O}}$ the corresponding semidirect product. A Lie groupoid $\mathcal{G} \rightrightarrows M$ is said to be source-locally trivial if the source map $s: \mathcal{G} \rightarrow M$ makes $\mathcal{G}$ into a locally trivial fibration. An orbit (or manifold) $\mathcal{O}$ is called of finite type if there is a proper map $f: \mathcal{O} \rightarrow \mathbb{R}$ with a finite number of critical points.

THEOREM 2.4 (Slice theorem [31]). - Let $\mathcal{G} \rightrightarrows M$ be a source-locally trivial proper Lie groupoid, and let $\mathcal{O}$ be an orbit of finite type of $\mathcal{G}$ on $M$. Then there is an invariant neighborhood $\mathcal{U}$ of $\mathcal{O}$ in $M$ such that the restriction $\mathcal{G}_{\mathcal{U}}$ of $\mathcal{G}$ to $\mathcal{U}$ is isomorphic to the restriction of $\mathcal{G}_{\mathcal{O}} \ltimes N_{\mathcal{O}}$ to a tubular neighborhood of the zero section in $N_{\mathcal{O}}$ (and also isomorphic to $\mathcal{G}_{\mathcal{O}} \ltimes N_{\mathcal{O}}$ itself).

We will now apply Theorem 2.3 to the case of symplectic and quasi-symplectic groupoids. Recall (see, e.g., [5,7]) that a symplectic groupoid is a Lie groupoid $\Gamma \rightrightarrows P$, where $\Gamma$ is equipped with a symplectic form $\omega$ such that the graph $\Delta=\{(p, q, p . q) \mid p, q \in \Gamma, s(p)=t(q)\}$ of the

$4^{\mathrm{e}}$ SÉRIE - TOME $39-2006-\mathrm{N}^{\circ} 5$ 
product operation of $\Gamma$ is a Lagrangian submanifold of $\Gamma \times \Gamma \times \bar{\Gamma}$, where $\bar{\Gamma}$ means $\Gamma$ with the opposite symplectic form $-\omega$. If $(\Gamma, \omega) \rightrightarrows P$ is a symplectic groupoid, then there is a unique Poisson structure $\Pi$ on $P$ such that the source map $s:(\Gamma, \omega) \rightarrow(P, \Pi)$ is Poisson and the target map $t:(\Gamma, \omega) \rightarrow(P, \Pi)$ is anti-Poisson; the path-connected components of the orbits of $\Gamma$ on $P$ are the symplectic leaves of $\Pi$. For example, consider the action groupoid $G \times \mathfrak{g}^{*} \rightrightarrows \mathfrak{g}^{*}$ of the coadjoint action of a Lie group $G$. Identify $G \times \mathfrak{g}^{*}$ with $T^{*} G$ via left translations, and equip it with the standard symplectic form. Then it becomes a symplectic groupoid, which we will call a standard symplectic groupoid and denote by $T^{*} G \rightrightarrows \mathfrak{g}^{*}$. The corresponding Poisson structure on $\mathfrak{g}^{*}$ is the standard linear (Lie-)Poisson structure.

It is easy to check that any sufficiently small slice of a (proper) symplectic groupoid is again a (proper) symplectic groupoid: the symplectic form of the slice is the restriction of the symplectic form of the original symplectic groupoid to the slice. A symplectic groupoid is called proper if it is proper as a Lie groupoid.

THEOREM 2.5. - Let $(\Gamma, \omega) \rightrightarrows(P, \Pi)$ be a proper symplectic groupoid with a fixed point $m \in P$. Then it is locally isomorphic (as a symplectic groupoid) to the standard symplectic groupoid $T^{*} G \rightrightarrows \mathfrak{g}^{*}$, where $G=G_{m}$ is the isotropy group of $m$. In other words, there are an invariant neighborhood $U$ of $m$ in $P$ and a neighborhood $V$ of 0 in $\mathfrak{g}^{*}$ invariant under the coadjoint action such that $((\Gamma, \omega) \rightrightarrows P)_{U} \cong\left(T^{*} G \rightrightarrows \mathfrak{g}^{*}\right)_{V}$.

In the above theorem, the isotropy group $G$ can be disconnected. The proof of Theorem 2.5 will be given in Section 2.6.

Consider now a quasi-symplectic groupoid in the sense of Xu [32] (also known as twisted presymplectic groupoid [3]). This is a Lie groupoid $\Gamma \rightrightarrows P$, equipped with a 2 -form $\omega$ on $\Gamma$ and a 3-form $\Omega$ on $P$, which satisfy the following four conditions:

(i) $\mathrm{d} \omega=t^{*} \Omega-s^{*} \Omega$.

(ii) $\mathrm{d} \Omega=0$.

(iii) The graph $\Delta=\{(p, q, p . q) \mid p, q \in \Gamma, s(p)=t(q)\}$ of the product operation of $\Gamma$ is isotropic with respect to the 2 -form $\omega \oplus \omega \oplus(-\omega)$ on $\Gamma \times \Gamma \times \Gamma$.

(iv) Identify $P$ with its unit section $\varepsilon(P)$ in $\Gamma$. Due to condition (iii), for each point $m \in P$, the differential $t_{*}$ of the target map $t$ can be restricted to a map

$$
t_{*}: \operatorname{ker} \omega_{m} \cap T_{m} s^{-1}(m) \rightarrow \operatorname{ker} \omega_{m} \cap T_{m} P
$$

(where ker $\omega_{m}$ denotes the kernel of $\omega$ at $m$ ), and the condition is that this restricted map is bijective.

The first three conditions mean that $\omega+\Omega$ is a 3-cocycle in the total de Rham complex of the groupoid $\Gamma \rightrightarrows P$ (see [32]), and the last condition is a weak nondegeneracy condition on $\omega$. If $\omega$ is nondegenerate and $\Omega=0$ then one gets back to the notion of symplectic groupoids. The base space of a quasi-symplectic groupoid is a manifold with a twisted Dirac structure (see [3]). It is easy to check that a sufficiently small slice of a (proper) quasi-symplectic groupoid is again a (proper) quasi-symplectic groupoid (we will leave it to the reader as an exercise).

Remark. - The convention on Lie groupoids used in this paper is different from [32]: our source map is the target map in [32] and vice versa.

A result of $\mathrm{Xu}$ [32, Proposition 4.8] says that if $(\Gamma \rightrightarrows P, \omega+\Omega)$ is a quasi-symplectic groupoid, and $\beta$ is an arbitrary 2 -form on $P$, then $\left(\Gamma \rightrightarrows P, \omega^{\prime}+\Omega^{\prime}\right)$, where $\omega^{\prime}=\omega+t^{*} \beta-s^{*} \beta$ and $\Omega^{\prime}=\Omega+\mathrm{d} \beta$, is again a quasi-symplectic groupoid, and moreover it is Morita-equivalent to $(\Gamma \rightrightarrows P, \omega+\Omega)$ - the notion of Morita equivalence of quasi-Hamiltonian groupoids will be recalled in Section 3.1. This result together with Theorem 2.5 immediately leads to the following: 
COROLlaRY 2.6. - If $(\Gamma \rightrightarrows P, \omega+\Omega)$ is a proper quasi-symplectic groupoid with a fixed point $m$, then it is locally isomorphic to a quasi-symplectic groupoid of the type $\left(T^{*} G \rightrightarrows \mathfrak{g}^{*}, \omega_{0}+t^{*} \beta-s^{*} \beta+\mathrm{d} \beta\right)$, where $\left(T^{*} G \rightrightarrows \mathfrak{g}^{*}, \omega_{0}\right)$ is the standard symplectic groupoid of the isotropy group $G=G_{m}$ of $m$, and $\beta$ is a 2 -form on $\mathfrak{g}^{*}$. In particular, $(\Gamma \rightrightarrows P, \omega+\Omega)$ is locally Morita equivalent to the standard symplectic groupoid $T^{*} G \rightrightarrows \mathfrak{g}^{*}$.

Proof. - Since $\Omega$ is a closed 3-form, locally it is exact, $\Omega=\mathrm{d} \beta$, and we can kill it by changing $\omega$ to $\omega_{0}=\omega+s^{*} \beta-t^{*} \beta$. In order to apply Theorem 2.5 , it remains to verify that $\omega_{0}$ is nondegenerate. At the fixed point $m$, the weak nondegeneracy condition is the same as the usual nondegeneracy condition, so $\omega_{0}$ is nondegenerate at $m$. The nondegeneracy of $\omega_{0}$ at $m$ implies the nondegeneracy of $\omega_{0}$ at the other points on the isotropy group $G_{m}$ via the compatibility condition (iii) of the definition, so $\omega_{0}$ is nondegenerate at $G_{m}$, and hence it is nondegenerate in a sufficiently small neighborhood of $G_{m}$ in $\Gamma$. In other words, if $B$ is a sufficiently small neighborhood of $m$ in $P$ then $\left(\left.\Gamma\right|_{B} \rightrightarrows B, \omega_{0}\right)$ will be a proper symplectic groupoid.

Recall that if $G$ is a connected compact Lie group, then the orbit space of $T^{*} G \rightrightarrows \mathfrak{g}^{*}$, i.e. the space of coadjoint orbits of $G$ on $\mathfrak{g}^{*}$ can be naturally identified with the corresponding closed Weyl chamber $\mathfrak{t}_{+}^{*}$. (Embed $\mathfrak{t}_{+}^{*} \subset \mathfrak{t}^{*}$ in $\mathfrak{g}^{*}$ in a natural way; then each coadjoint orbit of $G$ in $\mathfrak{g}^{*}$ will intersect $\mathfrak{t}_{+}^{*}$ at exactly one point.) However, if $G$ is disconnected, then the orbit space of $T^{*} G \rightrightarrows \mathfrak{g}^{*}$ is not necessarily $\mathfrak{t}_{+}^{*}$, but may be a quotient of $\mathfrak{t}_{+}^{*}$ by a finite group action. The reason is that, if $G$ is disconnected, then its coadjoint action on $\mathfrak{g}^{*}$ may mix the connected coadjoint orbits (orbits of the connected part $G^{0}$ of $G$ ) by an action of $G / G^{0}$. For example, consider the following disconnected double covering $G=\mathbb{T}^{2} \sqcup \theta . \mathbb{T}^{2}$ of $\mathbb{T}^{2}$, where $\theta$ is an element such that

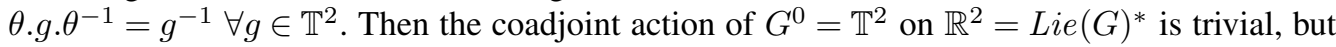
the coadjoint action of $\theta$ on $\mathbb{R}^{2}$ is given by the map $(x, y) \mapsto(-x,-y)$. The quotient space of $\mathbb{R}^{2}$ by the coadjoint action of $G$ is the orbifold $\mathbb{R}^{2} / \mathbb{Z}_{2}$. In order to avoid such orbifolds, we make the following definition:

DEFINITION 2.7. - We will say that a (not necessarily connected) compact Lie group is coadconnected if its coadjoint orbits are connected.

If a compact Lie group $G$ is coad-connected, then the orbit space of $T^{*} G \rightrightarrows \mathfrak{g}^{*}$ can be naturally identified with a Weyl chamber just like in the connected case. Of course, if $G$ is connected then it is automatically coad-connected.

COROLLARY 2.8. - If $(\Gamma \rightrightarrows P, \omega+\Omega)$ is a proper quasi-symplectic groupoid whose isotropy groups are coad-connected, then the orbit space $P / \Gamma$ is a manifold with locally polyhedral boundary: locally near each point it looks like a Weyl chamber of a compact Lie group.

If in the above corollary we drop the "coad-connected" condition, then we will have to replace "manifold" by "orbifold", and "Weyl chamber" by "quotient of a Weyl chamber by a finite group action".

\subsection{The averaging process}

Let us now proceed to the proof of Theorem 2.3. It will occupy the rest of Section 2. So from now on until the end of Section 2, we will denote by $\Gamma \rightrightarrows B$ a proper Lie groupoid with a fixed point $x_{0} \in B$, and by $G=G_{x_{0}}$ the compact isotropy group of $x_{0}$. A simple fact already observed by Weinstein [31] is that, due to the properness, any neighborhood of $x_{0}$ in $B$ will contain a closed ball-like neighborhood saturated by compact orbits of $\Gamma$. By shrinking $B$ if necessary, we can assume that $B$ is a closed ball, the orbits on $B$ are compact, and the source map $s: \Gamma \rightarrow B$ is a trivial fibration.

$4^{\text {e }}$ SÉRIE - TOME $39-2006-\mathrm{N}^{\circ} 5$ 
Note that Theorem 2.3 is essentially equivalent to the existence of a smooth surjective homomorphism $\phi$ from $\Gamma$ to $G$ (after shrinking $B$ to a sufficiently small invariant neighborhood of $x_{0}$ ), i.e. a smooth map $\phi: \Gamma \rightarrow G$ which satisfies

$$
\phi(p . q)=\phi(p) . \phi(q) \quad \forall(p, q) \in \Gamma_{(2)}:=\{(p, q) \in \Gamma \times \Gamma, s(p)=t(q)\},
$$

and such that the restriction of $\phi$ to $G=s^{-1}\left(x_{0}\right) \subset \Gamma$ is an automorphism of $G$. We may assume that this automorphism is identity.

Indeed, if there is an isomorphism from $\Gamma \rightrightarrows B$ to an action groupoid $G \ltimes U$, then the composition of the isomorphism map $\Gamma \rightarrow G \times U$ with the projection $G \times U \rightarrow G$ is such a homomorphism. Conversely, we have:

Lemma 2.9. - Assume that there is a homomorphism $\phi: \Gamma \rightarrow G$, whose restriction to $G=$ $s^{-1}\left(x_{0}\right) \subset \Gamma$ is the identity map of $G$. Then $\Gamma$ is locally linearizable.

Proof. - Shrinking $B$ to a sufficiently small invariant neighborhood of $z$ in $B$ if necessary, we get a diffeomorphism

$$
(\phi, s): \Gamma \rightarrow G \times B .
$$

Denote by $\theta$ the inverse map of $(\phi, s)$. Then there is an action of $G$ on $B$ defined by $g . x=$ $t(\theta(g, x))$, and the map $(\phi, s)$ will be an isomorphism from $\Gamma \rightrightarrows B$ to the action groupoid $G \ltimes B$. This action groupoid is linearizable by the classical Bochner's theorem [2], implying that the groupoid $\Gamma \rightrightarrows B$ is linearizable.

The above lemma reduces the problem of linearizing $\Gamma$ to the problem of finding a homomorphism from $\Gamma$ to $G$ which extends the identity map of $G$. In order to find such a homomorphism, we will use the averaging method. The idea is to start from an arbitrary smooth map $\phi: \Gamma \rightarrow G$ such that $\left.\phi\right|_{G}=$ Id. (Recall that $G=s^{-1}\left(x_{0}\right)=t^{-1}\left(x_{0}\right)$.) Then equality (2.2) is not satisfied in general, but it is satisfied for $p, q \in G$. Hence it is "nearly satisfied" in a small neighborhood of $G=s^{-1}\left(x_{0}\right)$ in $\Gamma$. In other words, if the base $B$ is small enough, then $\phi(p . q) \phi(q)^{-1}$ is near $\phi(p)$ for any $(p, q) \in \Gamma_{(2)}$. We will replace $\phi(p)$ by the average value of $\phi(p . q) \phi(q)^{-1}$ for $q$ running on $t^{-1}(s(p))$ (it is to be made precise how to define this average value). This way we obtain a new map $\widehat{\phi}: \Gamma \rightarrow G$, which will be shown to be "closer" to a homomorphism than the original map $\phi$. By iterating the process and taking the limit, we will obtain a true homomorphism $\phi_{\infty}$ from $\Gamma$ to $G$.

Notice that the $t$-fibers of $\Gamma \rightrightarrows B$ are compact and diffeomorphic to $G=t^{-1}\left(x_{0}\right)$ by assumptions. As a consequence, there exists a smooth Haar probability system $\left(\mu_{x}\right)$ on $\Gamma$, i.e. a smooth Haar system such that for each $x \in B$, the volume of $t^{-1}(x)$ with respect to $\mu_{x}$ is 1 . Such a Haar probability system $\left(\mu_{x}\right)$ can be constructed as follows: begin with an arbitrary Haar system $\left(\mu_{x}^{\prime}\right)$ on $\Gamma$, then define $\mu=\mu^{\prime} / I$ where $I$ is the left-invariant function $I(g)=\int_{t^{-1}(t(g))} \mathrm{d} \mu_{t(g)}^{\prime}$. We will fix a Haar probability system $\mu=\left(\mu_{x}\right)$ on $\Gamma$.

We fix an ad-invariant metric on the Lie algebra $\mathfrak{g}$ of $G$ and the induced bi-invariant metric $d$ on $G$ itself. Denote by $1_{G}$ the neutral element of $G$. For each number $\rho>0$, denote by $B_{\mathfrak{g}}(\rho)$ (resp. $B_{G}(\rho)$ ) the closed ball of radius $\rho$ in $\mathfrak{g}$ (resp. $G$ ) centered at 0 (resp. $1_{G}$ ). By resizing the metric if necessary, we will assume that the exponential map

$$
\exp : B_{\mathfrak{g}}(1) \rightarrow B_{G}(1)
$$

is a diffeomorphism. Denote by

$$
\log : B_{G}(1) \rightarrow B_{\mathfrak{g}}(1)
$$


the inverse of exp. Define the distance $\Delta(\phi)$ of $\phi: \Gamma \rightarrow G$ from being a homomorphism as follows:

$$
\Delta(\phi)=\sup _{(p, q) \in \Gamma_{(2)}} d\left(\phi(p \cdot q) \cdot \phi(q)^{-1} \cdot \phi(p)^{-1}, 1_{G}\right) .
$$

Let $\phi: \Gamma \rightarrow G$ be a smooth map such that $\left.\phi\right|_{G}$ is identity. We will assume that $\Delta(\phi) \leqslant 1$, so that the following map $\widehat{\phi}: \Gamma \rightarrow G$ is clearly well-defined:

$$
\widehat{\phi}(p)=\exp \left(\int_{q \in t^{-1}(s(p))} \log \left(\phi(p \cdot q) \cdot \phi(q)^{-1} \cdot \phi(p)^{-1}\right) \mathrm{d} \mu_{s(p)}\right) \cdot \phi(p) .
$$

Since $\mu$ is invariant under left translations, by the change of variable $r=p . q$, we can also write $\widehat{\phi}$ as:

$$
\widehat{\phi}(p)=\exp \left(\int_{r \in t^{-1}(t(p))} \log \left(\phi(r) \cdot \phi\left(p^{-1} \cdot r\right)^{-1} \cdot \phi(p)^{-1}\right) \mathrm{d} \mu_{t(p)}\right) \cdot \phi(p) .
$$

Due to the commutativity of the maps exp and log with the adjoint actions, we can also write $\widehat{\phi}$ as follows:

$$
\widehat{\phi}(p)=\phi(p) \cdot \exp \left(\int_{q \in t^{-1}(s(p))} \log \left(\phi(p)^{-1} \cdot \phi(p \cdot q) \cdot \phi(q)^{-1}\right) \mathrm{d} \mu_{s(p)}\right) .
$$

It is clear that $\widehat{\phi}$ is a smooth map from $\Gamma$ to $G$, and its restriction to $G=s^{-1}(0) \subset \Gamma$ is also identity. The proof of the following lemma, which says that when $G$ is essentially Abelian we are done, is straightforward (we will not need this lemma in the proof of Theorem 2.3, so we will omit its proof here):

LEMMA 2.10. - With the above notations, if $G$ is essentially commutative (i.e. the connected component of the neutral element of $G$ is commutative) then $\widehat{\phi}$ is a homomorphism.

In general, due to the non-commutativity of $G, \widehat{\phi}$ is not necessarily a homomorphism, but $\Delta(\widehat{\phi})$ (the distance of $\widehat{\phi}$ from being a homomorphism) is of the order of $\Delta(\phi)^{2}$ (Lemma 2.12). It means that we have the following fast convergent iterative process: starting from an arbitrary given smooth map $\phi: \Gamma \rightarrow G$, such that $\left.\phi\right|_{G}=\mathrm{Id}$, construct a sequence of maps $\phi_{n}: \Gamma \rightarrow G$ by the recurrence formula $\phi_{1}=\phi, \phi_{n+1}=\widehat{\phi_{n}}$.

In the next subsections we will show that this sequence is well-defined (after shrinking $B$ once to a smaller invariant neighborhood of $x_{0}$ if necessary), and that

$$
\phi_{\infty}=\lim _{n \rightarrow \infty} \phi_{n}
$$

exists, is smooth, and is a homomorphism from $\Gamma$ to $G$.

Remark. - The above iterative averaging process is inspired by a similar process which was employed by Grove, Karcher and Ruh in [12] to prove that near-homomorphisms between compact Lie groups can be approximated by homomorphisms. The idea of using GroveKarcher-Ruh's iterative averaging method was proposed by Weinstein [28,29,31], though he looked at the "wrong" map: he considered near-homomorphisms from $G$ to the group of bisections of $\Gamma \rightrightarrows B$ instead of near-homomorphisms from $\Gamma$ to $G$, and was not able to prove the convergence of a corresponding iterative averaging process.

$4^{\mathrm{e}}$ SÉRIE - TOME $39-2006-\mathrm{N}^{\circ} 5$ 


\subsection{Spaces of maps and $C^{k}$-norms}

This is an auxiliary subsection where we fix some notations and write down some standard useful inequalities.

For each $n \in \mathbb{N}$, the space of composable $n$-tuples

$$
\Gamma_{(n)}:=\left\{\left(p_{1}, \ldots, p_{n}\right) \in \Gamma \times \cdots \times \Gamma \mid s\left(p_{i}\right)=t\left(p_{i+1}\right) \forall i<n\right\}
$$

is smoothly diffeomorphic to $B \times G \times \cdots \times G$ ( $n$ copies of $G$ ). To fix the norms, we will fix such a diffeomorphism (of the same smoothness class as $\Gamma$ ) for each $n$. We will mainly use the manifolds $B, \Gamma=\Gamma_{(1)}, \Gamma_{(2)}$ and $\Gamma_{(3)}$. To fix the norms on $B$ (i.e. for maps from and to $B$ ), we will assume that $B$ is a closed ball centered at $z$ in a given Euclidean space. (We will shrink $B$ whenever necessary, but the norm of the Euclidean space which contains it will not be changed.)

If $V_{1}$ and $V_{2}$ are two nonnegative numbers which depend on several variables and parameters, then we will write $V_{1} \preceq V_{2}$ (read $V_{1}$ is smaller than $V_{2}$ up to a multiplicative constant) if there is a positive constant $C$ (which does not depend on the variables of $V_{1}$ and $V_{2}$, though it may depend some fixed parameters) such that $V_{1} \leqslant C V_{2}$. We can also write $V_{1}=O\left(V_{2}\right)$ using Landau notation. We will write $V_{1} \approx V_{2}$ if $V_{1} \preceq V_{2} \preceq V_{1}$.

We are interested in the $C^{k}$-topology ( $k \leqslant m$ if $\Gamma$ is only $C^{m}$-smooth) of the spaces of maps from $B, \Gamma, \Gamma_{(2)}, \Gamma_{(3)}$ to $\mathfrak{g}$ and $G$. We will use $\|\cdot\|_{k}$ to denote a chosen $C^{k}$-norm on the vector space of $C^{k}$-functions from $N$ to $\mathfrak{g}$, where $N$ denotes one of the spaces $B, \Gamma, \Gamma_{(2)}, \Gamma_{(3)}$, etc. (Recall that $B$ is a closed ball, so $N$ is compact with boundary. It does not matter much which $C^{k}$-norm we choose, as long as it is a norm which provides the $C^{k}$-topology.) We will need the following inequalities:

If $f_{r}$ is a family of functions from $N$ to $\mathfrak{g}$ which depends on a parameter $r$ which lives in a probability space $R$ then, due to the triangular inequality for a norm, we have

$$
\left\|\int_{R} f_{r} \mathrm{~d} r\right\|_{k} \leqslant \sup _{r \in R}\left\|f_{r}\right\|_{k} .
$$

For $f_{1}, f_{2}: N \rightarrow \mathfrak{g}$, assuming that $\log \left(\exp \left(f_{1}\right) \cdot \exp \left(f_{2}\right)\right)$ is well-defined, we have

$$
\left\|\log \left(\exp \left(f_{1}\right) \cdot \exp \left(f_{2}\right)\right)-f_{1}-f_{2}\right\|_{0} \preceq\left\|f_{1}\right\|_{0}\left\|f_{2}\right\|_{0},
$$

and

$$
\left\|\log \left(\exp \left(f_{1}\right) \cdot \exp \left(f_{2}\right)\right)\right\|_{0} \preceq\left\|f_{1}+f_{2}\right\|_{0} .
$$

If, moreover, $\left\|f_{1}\right\|_{k-1},\left\|f_{2}\right\|_{k-1} \preceq 1$ (for some fixed $k \geqslant 1$ ) then

$$
\begin{aligned}
& \left\|\log \left(\exp \left(f_{1}\right) \cdot \exp \left(f_{2}\right)\right)-f_{1}-f_{2}\right\|_{k} \\
& \quad \preceq\left\|f_{1}\right\|_{0}\left\|f_{2}\right\|_{k}+\left\|f_{2}\right\|_{0}\left\|f_{1}\right\|_{k}+\left\|f_{1}\right\|_{k-1}+\left\|f_{2}\right\|_{k-1},
\end{aligned}
$$

and

$$
\begin{aligned}
& \left\|\log \left(\exp \left(f_{1}\right) \cdot \exp \left(f_{2}\right)\right)\right\|_{k} \\
& \quad \preceq\left\|f_{1}+f_{2}\right\|_{k}+\left\|f_{1}\right\|_{0}\left\|f_{2}\right\|_{k}+\left\|f_{2}\right\|_{0}\left\|f_{1}\right\|_{k}+\left\|f_{1}\right\|_{k-1}+\left\|f_{2}\right\|_{k-1} .
\end{aligned}
$$

The above inequalities follow directly from the Leibniz rule of derivation and the fact that the map $K: \mathfrak{g} \times \mathfrak{g} \rightarrow \mathfrak{g}$ defined by $K(x, y)=\log (\exp (x) . \exp (y))-x-y$ (this map is well-defined 
in a neighborhood of the origin in $\mathfrak{g} \times \mathfrak{g}$ ) is an analytic map with the following properties: the linear part of $K(x, y)$ is trivial, the quadratic part of $K$ contains only terms of the type $x_{i} y_{j}$ (where $\left(x_{i}\right)$ and $\left(y_{i}\right)$ are the coordinates of $x$ and $y$ respectively), and $K(x, y)=0$ on the subspaces $\{x=0\},\{y=0\}$ and $\{x+y=0\}$. Let us prove, for example, inequality (2.15). We mainly have to estimate terms of the type $\partial_{z}^{k} K\left(f_{1}(z), f_{2}(z)\right)$, where $z$ denotes an element of $N$, and $\partial_{z}^{k}$ denotes a $k$-times partial derivative in $z$ (in some local coordinate system). By the Leibniz rule, $\partial_{z}^{k} K\left(f_{1}(z), f_{2}(z)\right)$ is a sum of terms of the following types: (i) $\partial_{f_{1}} K\left(f_{1}, f_{2}\right) \partial_{z}^{k} f_{1}$; (ii) $\partial_{f_{2}} K\left(f_{1}, f_{2}\right) \partial_{z}^{k} f_{2}$; (iii) products of partial derivatives which are of order $\leqslant k-1$ in $z$. Note that $\left|\partial_{x} K(x, y)\right| \preceq|y|$ because $\partial_{x} K(x, 0)=0$, and therefore the terms of type (i) can be majored by $\left\|f_{2}\right\|_{0}\left\|f_{1}\right\|_{k}$ (up to a multiplicative constant). Similarly, the terms of type (ii) can be majored by $\left\|f_{1}\right\|_{0}\left\|f_{2}\right\|_{k}$. On the other hand, in a product of type (iii), we can major one factor by $\left\|f_{1}\right\|_{k-1}+\left\|f_{2}\right\|_{k-1}$ and the other factors by constants (because we assumed that $\left\|f_{1}\right\|_{k-1},\left\|f_{2}\right\|_{k-1} \preceq 1$ ), so every term of type (iii) can be majored by $\left\|f_{1}\right\|_{k-1}+\left\|f_{2}\right\|_{k-1}$. Summing up all the above terms together, we obtain inequality (2.15).

The space $C^{k}(N, G)$ of $C^{k}$-maps from $N$ to $G$ is not a vector space, but rather a Banach Lie group modeled on $C^{k}(N, \mathfrak{g})$. We will denote by $1_{G}$ the neutral element of this group, i.e. the map from $N$ to $G$ which sends every element of $N$ to the neutral element of $G$, also denoted by $1_{G}$. If $f: N \rightarrow G$ is a continuous function then we put

$$
\|f\|_{0}:=\sup _{x \in N} d\left(f(x), 1_{G}\right)
$$

where $d(\cdot, \cdot)$ is the metric on $G$. If $f: N \rightarrow G$ is a $C^{k}$-map $(k \geqslant 1)$ such that $\|f\|_{0} \leqslant 1$ (so that $\log (f): N \rightarrow \mathfrak{g}$ is well defined), we put

$$
\|f\|_{k}:=\|\log (f)\|_{k}
$$

and call it the $C^{k}$-norm of $f$ by abuse of language. What it measures is a $C^{k}$-distance from $f$ to the neutral map $1_{G}$. In particular, $\left\|1_{G}\right\|_{k}=0 \forall k$. We will not need $\|f\|_{k}(k \geqslant 1)$ when $\|f\|_{0}>1$, but let us put $\|f\|_{k}:=2(k \geqslant 1)$ whenever $\|f\|_{0}>1$, so that the expression $\|f\|_{k}$ makes sense for all $f \in C^{k}(N, G)$.

Let us write down some other useful standard inequalities, whose proof is similar, if not simpler, to the proof of inequality (2.15).

If $\chi$ is a given smooth map from $N$ to $N^{\prime}$ (e.g., the product map from $\Gamma_{(2)}$ to $\Gamma$ ) and $f$ is a map from $N^{\prime}$ to $\mathfrak{g}$ or $G$, then we have (for each fixed nonnegative integer $k$ which does not exceed the smoothness class of the groupoid):

$$
\|f \circ \chi\|_{k} \preceq\|f\|_{k} .
$$

If $f_{1}, f_{2}$ are two functions from $N$ to $G$ such that $\left\|f_{1}\right\|_{0},\left\|f_{2}\right\|_{0} \leqslant 1$ then

$$
\left\|f_{1} \cdot f_{2}\right\|_{0} \preceq\left\|f_{1}\right\|_{0}+\left\|f_{2}\right\|_{0}
$$

and if moreover $\left\|f_{1}\right\|_{k-1},\left\|f_{2}\right\|_{k-1} \preceq 1$ (for some fixed $k \geqslant 1$ ), then we have:

$$
\left\|f_{1} \cdot f_{2}\right\|_{k} \preceq\left\|f_{1}\right\|_{k}+\left\|f_{2}\right\|_{k}
$$

and (more refined inequalities)

$$
\left\|f_{1} \cdot f_{2}\right\|_{k}-\left\|f_{2}\right\|_{k} \preceq\left\|f_{1}\right\|_{k}+\left\|f_{1}\right\|_{0}\left\|f_{2}\right\|_{k}
$$

$4^{\text {e }}$ SÉRIE - TOME $39-2006-\mathrm{N}^{\circ} 5$ 


$$
\left\|f_{1} \cdot f_{2} \cdot f_{1}^{-1}\right\|_{k} \preceq\left\|f_{2}\right\|_{k}+\left\|f_{1}\right\|_{k} \cdot\left\|f_{2}\right\|_{0} .
$$

Finally, we will need the following result about Cauchy sequences in Banach Lie groups, which we will state as a lemma:

Lemma 2.11. - Suppose that $f_{n}(n \in \mathbb{N})$ are maps from $N$ to $G$ such that $\sum_{n=1}^{\infty}\left\|f_{n}\right\|_{k}$ converges (for some given nonnegative integer $k$ which does not exceed the smoothness class of $N)$. Then the product $f_{n} . f_{n-1} \cdots \cdot f_{1}$ converges in the $C^{k}$-topology when $n \rightarrow \infty$ to a $C^{k}$-map from $N$ to $G$.

\section{4. $C^{0}$-estimates}

LEMMA 2.12. - For any $\phi: \Gamma \rightarrow G$ with $\Delta(\phi) \leqslant 1$ we have

$$
\Delta(\widehat{\phi}) \preceq(\Delta(\phi))^{2} .
$$

In particular, there is a positive constant $C_{0}>0, C_{0} \leqslant 1$ such that if $\Delta(\phi) \leqslant C_{0}$ then $\widehat{\phi}$ is welldefined and

$$
\Delta(\widehat{\phi}) \leqslant(\Delta(\phi))^{2} / C_{0} \leqslant \Delta(\phi)
$$

Proof. - Denote

$$
\psi(p, q)=\phi(p . q) . \phi(q)^{-1} . \phi(p)^{-1}
$$

and

$$
\widehat{\psi}(p, q)=\widehat{\phi}(p \cdot q) \cdot \widehat{\phi}(q)^{-1} . \widehat{\phi}(p)^{-1} \text {. }
$$

Then $\psi$ and $\widehat{\psi}$ are functions from $\Gamma_{(2)}$ to $G$. By definition of $\widehat{\phi}$, we have

$$
\begin{aligned}
\widehat{\psi}(p, q)= & \exp \left(\int_{r \in T} \log (\psi(p \cdot q, r)) \mathrm{d} \mu\right) \cdot \phi(p \cdot q) \cdot \phi(q)^{-1} \\
& . \exp \left(\int_{r \in T}^{-1} \log (\psi(q, r)) \mathrm{d} \mu\right)^{-1} \\
& . \phi(p)^{-1} \exp \left(\int_{r^{\prime} \in t^{-1}(s(p))} \log \left(\psi\left(p, r^{\prime}\right)\right) \mathrm{d} \mu\right)^{-1} \\
= & \phi(p \cdot q) \cdot \phi(q)^{-1} \cdot \phi(p)^{-1} \cdot E(p, q)=\psi(p, q) \cdot E(p, q),
\end{aligned}
$$

where $T=t^{-1}(s(q))$ and

$$
\begin{aligned}
E(p, q)= & A d_{\psi(p, q)^{-1}} \exp \left(\int_{r \in T(s(q))} \log (\psi(p . q, r)) \mathrm{d} \mu\right) \\
& . A d_{\phi(p)} \exp \left(\int_{r \in T(s(q))}^{-1} \log (\psi(q, r)) \mathrm{d} \mu\right)^{-1} \\
& . \exp \left(\int_{r^{\prime} \in t^{-1}(s(p))} \log \left(\psi\left(p, r^{\prime}\right)\right) \mathrm{d} \mu\right)^{-1}
\end{aligned}
$$

ANNALES SCIENTIFIQUES DE L'ÉCOLE NORMALE SUPÉRIEURE 


$$
\begin{aligned}
= & \exp \left(\int_{r \in T} \log \left(\psi(p, q)^{-1} \cdot \psi(p \cdot q, r) \cdot \psi(p, q)\right) \mathrm{d} \mu\right) \\
& . \exp \left(\int_{r \in T} \log \left(\phi(p) \cdot \psi(q, r)^{-1} \cdot \phi(p)^{-1}\right) \mathrm{d} \mu\right) \\
& . \exp \left(\int_{r \in T} \log \left(\psi(p, q \cdot r)^{-1}\right) \mathrm{d} \mu\right) \quad\left(\text { we replaced } r^{\prime} \text { by } r=q^{-1} \cdot r^{\prime}\right) \\
= & \exp \left(\int_{r \in T} \log \left(A_{1}\right) \mathrm{d} \mu\right) \cdot \exp \left(\int_{r \in T} \log \left(A_{2}\right) \mathrm{d} \mu\right) \cdot \exp \left(\int_{r \in T} \log \left(A_{3}\right) \mathrm{d} \mu\right),
\end{aligned}
$$

where

$$
\begin{aligned}
& A_{1}=\psi(p, q)^{-1} \cdot \psi(p \cdot q, r) \cdot \psi(p, q), \\
& A_{2}=\phi(p) \cdot \psi(q, r)^{-1} \cdot \phi(p)^{-1} \\
& A_{3}=\psi(p, q \cdot r)^{-1} .
\end{aligned}
$$

One verifies directly that

$$
A_{1} \cdot A_{2} \cdot A_{3}=\psi(p \cdot q)^{-1} \text {. }
$$

Consider $A_{1}, A_{2}, A_{3}$ as maps from $\Gamma_{3}$ to $G$. By definition, $\Delta(\phi)=\|\psi\|_{0}$. The inequality $\Delta(\phi) \leqslant 1$ in the hypothesis of Lemma 2.12, together with the fact that the metric on $G$ is biinvariant, implies that

$$
\left\|A_{1}\right\|_{0}=\left\|A_{2}\right\|_{0}=\left\|A_{3}\right\|_{0}=\|\psi\|_{0}=\Delta(\phi) \leqslant 1 .
$$

Applying inequalities (2.13), (2.12) and (2.32) several times to $E(p, q)$, we get:

$$
\begin{aligned}
\log E(p, q) & =\varepsilon_{1}+\int_{r \in T} \log \left(A_{1}\right) \mathrm{d} \mu+\int_{r \in T} \log \left(A_{2}\right) \mathrm{d} \mu+\int_{r \in T} \log \left(A_{3}\right) \mathrm{d} \mu \\
& =\varepsilon_{1}+\int_{r \in T}\left[\log \left(A_{1}\right)+\log \left(A_{2}\right)+\log \left(A_{3}\right)\right] \mathrm{d} \mu \\
& =\varepsilon_{1}+\varepsilon_{2}+\int_{r \in T} \log \left(A_{1} A_{2} A_{3}\right) \mathrm{d} \mu \\
& =\varepsilon_{1}+\varepsilon_{2}-\log (\psi(p, q)),
\end{aligned}
$$

where $T=t^{-1}(s(q))$ and $\varepsilon_{1}$ and $\varepsilon_{2}$ are some functions such that

$$
\left\|\varepsilon_{1}\right\|_{0},\left\|\varepsilon_{2}\right\|_{0} \preceq \Delta(\phi)^{2} .
$$

In other words, we have $\|\log (\psi(p, q))+\log E(p, q)\|_{0} \preceq \Delta(\phi)^{2}$, which implies, by inequality (2.14), that $\|\psi \cdot E\|_{0} \preceq \Delta(\phi)^{2}$. But we have $\widehat{\psi}(p, q)=\psi(p, q)$. $E(p, q)$, therefore

$$
\Delta(\widehat{\phi})=\|\widehat{\psi}\|_{0}=\|\psi \cdot E\| \preceq \Delta(\phi)^{2} .
$$

Lemma 2.12 immediately implies the uniform convergence (i.e. convergence in the $C^{0}$ topology) of the sequence of maps $\phi_{n}: \Gamma \rightarrow G$, defined iteratively by $\phi_{n+1}=\widehat{\phi_{n}}$, beginning $4^{\text {e }}$ SÉRIE - TOME $39-2006-\mathrm{N}^{\circ} 5$ 
with an arbitrary smooth map $\phi_{1}$ which satisfies the inequality $\Delta\left(\phi_{1}\right) \leqslant C_{0} / 4$. (This inequality can always be achieved by shrinking $B$ if necessary.) Indeed, since $\left\|\psi_{2}\right\|_{0}=\Delta\left(\phi_{2}\right) \leqslant$ $\left(\Delta\left(\phi_{1}\right)\right)^{2} / C_{0} \leqslant \Delta\left(\phi_{1}\right) \leqslant C_{0} / 4 \leqslant 1 / 4$ by Lemma 2.12 , where

$$
\psi_{n}(p, q)=\phi_{n}(p \cdot q) \cdot \phi_{n}(q)^{-1} \cdot \phi_{n}(p)^{-1},
$$

we can define $\phi_{2}=\widehat{\phi_{1}}$, and so on, hence $\phi_{n}$ is well defined for all $n \in \mathbb{N}$. By recurrence on $n$, one can show easily that we have

$$
\left\|\psi_{n}\right\|_{0} \leqslant C_{0} \cdot\left(b_{0}\right)^{2^{n}} \quad \forall n \in \mathbb{N}, \quad \text { where } b_{0}=\frac{1}{2}<1,
$$

which implies in particular that $\sum_{n=1}^{\infty}\left\|\psi_{n}\right\|_{0}<\infty$ (this is a very fast converging series). Put

$$
\Psi_{n}(p)=\exp \left(\int_{q \in t^{-1}(s(p))} \log \left(\psi_{n}(p \cdot q)\right) \mathrm{d} \mu\right) .
$$

Then $\left\|\Psi_{n}\right\|_{0} \preceq\left\|\psi_{n}\right\|_{0}$ (by inequalities (2.19) and (2.12)), which together with $\sum_{n=1}^{\infty}\left\|\psi_{n}\right\|_{0}<\infty$ implies that

$$
\sum_{n=1}^{\infty}\left\|\Psi_{n}\right\|_{0}<\infty
$$

This last inequality implies the convergence of the product $\Psi_{n} \cdot \Psi_{n-1} \cdot \cdots, \Psi_{1}$ in the $C^{0}$-topology when $n \rightarrow \infty$. But

$$
\Psi_{n} . \Psi_{n-1} \cdot \cdots \cdot \Psi_{1}=\phi_{n+1} \cdot \phi_{1}^{-1} .
$$

Thus $\phi_{n}$ converges in the $C^{0}$-topology when $n \rightarrow \infty$. Denote by $\phi_{\infty}$ the limit

$$
\phi_{\infty}=\lim _{n \rightarrow \infty} \phi_{n}
$$

Then $\phi_{\infty}$ is a continuous homomorphism from $\Gamma$ to $G$. It is also clear that the restriction of $\phi_{\infty}$ to $G$ is the identity map from $G$ to itself.

It remains to show that $\phi_{\infty}$ is smooth. This is the purpose of the next subsection, where we will show that for any $k \in \mathbb{N}, k \leqslant m$ if $\Gamma$ belongs to the class $C^{m}$ only, we have $\phi_{\infty}=\lim _{n \rightarrow \infty} \phi_{n}$ in the $C^{k}$-topology as well.

\section{5. $C^{k}$-estimates}

Roughly speaking, we want to estimate $\psi_{n}$ in order to show that, if $k$ does not exceed the smoothness class of the groupoid $\Gamma \rightrightarrows B$, then $\sum_{n=1}^{\infty}\left\|\psi_{n}\right\|_{k}<\infty$. If this series converges, then similarly to the previous subsection, we also have $\sum_{n=1}^{\infty}\left\|\Psi_{n}\right\|_{k}<\infty$ where $\Psi_{n}=\phi_{n+1}$. $\phi_{n}^{-1}$ is given by formula (2.38), hence the product $\Psi_{n} . \Psi_{n-1} \cdot \cdots \cdot \Psi_{1}$ converges in the $C^{k}$-topology when $n \rightarrow \infty$, implying that $\phi_{n} \rightarrow \phi_{\infty}$ in the $C^{k}$-topology.

LEMMA 2.13. - Let $k \in \mathbb{N}$ be a natural number which does not exceed the smoothness class of the groupoid $\Gamma \rightrightarrows B$. Assume that $\|\psi\|_{0}=\Delta(\phi) \leqslant 1$ and $\|\phi\|_{k-1} \preceq 1$. Then we have:

$$
\|\widehat{\psi}\|_{k} \preceq\|\psi\|_{0}\|\psi\|_{k}+\|\psi\|_{k-1}+\|\psi\|_{0}\|\phi\|_{k-1}+\|\psi\|_{0}^{2}\|\phi\|_{k} .
$$


Proof. - Assume that $\|\phi\|_{k-1} \preceq 1$ by hypothesis of Lemma 2.13. Then by inequalities (2.21) and (2.19), we have $\|\psi\|_{k-1} \preceq\|\phi\|_{k-1} \preceq 1$. Let $A_{1}, A_{2}, A_{3}$ be the functions defined by Eq. (2.30). We want to estimate them. For $A_{3}=\psi\left(p, q \cdot r^{-1}\right)^{-1}$, using inequality (2.19), we get:

$$
\left\|A_{3}\right\|_{k-1} \preceq\|\psi\|_{k-1} \preceq 1 \quad \text { and } \quad\left\|A_{3}\right\|_{k} \preceq\|\psi\|_{k} .
$$

For $A_{1}=\psi(p, q)^{-1} . \psi(p . q, r) . \psi(p, q)$, using inequality (2.21) (and inequality (2.19)), we also get

$$
\left\|A_{1}\right\|_{k-1} \preceq\|\psi\|_{k-1} \preceq 1 \quad \text { and } \quad\left\|A_{1}\right\|_{k} \preceq\|\psi\|_{k} .
$$

The estimation of $A_{2}=\phi(p) . \psi(q, r)^{-1} . \phi(p)^{-1}$ is more complicated, because it involves the function $\phi$ directly. Using inequality (2.23) we get

$$
\left\|A_{2}\right\|_{k-1} \preceq\|\psi\|_{k-1}+\|\phi\|_{k-1}\|\psi\|_{0} \preceq 1
$$

and

$$
\left\|A_{2}\right\|_{k} \preceq\|\psi\|_{k}+\|\phi\|_{k}\|\psi\|_{0} .
$$

Applying inequality (2.15) and the above inequalities to $E(p, q)$, we get that

$$
\left\|\varepsilon_{1}\right\|_{k},\left\|\varepsilon_{2}\right\|_{k} \preceq\left(\|\psi\|_{k}+\|\phi\|_{k}\|\psi\|_{0}\right)\|\psi\|_{0}+\left(\|\psi\|_{k-1}+\|\phi\|_{k-1}\|\psi\|_{0}\right) .
$$

Moreover, we have

$$
\|E\|_{i} \preceq\|\psi\|_{i}+\|\psi\|_{0}\|\phi\|_{i} \quad \forall i=0, \ldots, k .
$$

Now applying inequality (2.16) and the last two inequalities, we get

$$
\begin{aligned}
\|\widehat{\psi}\|_{k} & =\|\exp (\log (\psi)) \cdot \exp (\log (E))\|_{k} \\
& \preceq\|\log (\psi)+\log (E)\|_{k}+\|\psi\|_{0}\|E\|_{k}+\|\psi\|_{k}\|E\|_{0}+\|\psi\|_{k-1}+\|E\|_{k-1} \\
& =\left\|\varepsilon_{1}+\varepsilon_{2}\right\|_{k}+\|\psi\|_{0}\|E\|_{k}+\|\psi\|_{k}\|E\|_{0}+\|\psi\|_{k-1}+\|E\|_{k-1} \\
& \preceq\|\psi\|_{0}\|\psi\|_{k}+\|\psi\|_{k-1}+\|\psi\|_{0}\|\phi\|_{k-1}+\|\psi\|_{0}^{2}\|\phi\|_{k} .
\end{aligned}
$$

LEMMA 2.14. - With the assumptions of Lemma 2.13 we have:

$$
\|\widehat{\phi}\|_{k}-\|\phi\|_{k} \preceq\|\psi\|_{k}+\|\psi\|_{0}\|\phi\|_{k} .
$$

Proof. - Applying inequality (2.22) to $\widehat{\phi}=\Psi$. $\phi$, we get

$$
\|\widehat{\phi}\|_{k}-\|\phi\|_{k} \preceq\|\Psi\|_{k}+\|\Psi\|_{0}\|\phi\|_{k} .
$$

Now replace $\|\Psi\|_{0}$ by $\|\psi\|_{0}$ and $\|\Psi\|_{k}$ by $\|\psi\|_{k}$.

LEMMA 2.15. - Assume that $\phi_{1}$ is a map from $\Gamma$ to $G$ such that $\Delta\left(\phi_{1}\right)<C_{0} / 4$, and that $\phi_{n+1}=\widehat{\phi_{n}}$ for any $n \in \mathbb{N}$, as in the previous subsection. Let $k$ be a natural number which does not exceed the smoothness class of the groupoid $\Gamma \rightrightarrows B$. Then there is a finite positive number $D_{k}>0$ and a positive number $0<b_{k}<1$, such that for any $n \in \mathbb{N}$ the following two inequalities hold:

$$
\left\|\phi_{n}\right\|_{k} \leqslant D_{k} \cdot\left(1-2^{-n}\right)
$$

$4^{\mathrm{e}}$ SÉRIE - TOME $39-2006-\mathrm{N}^{\circ} 5$ 
and

$$
\left\|\psi_{n}\right\|_{k} \leqslant D_{k} \cdot\left(b_{k}\right)^{2^{n}} .
$$

Proof. - We will prove the above lemma by induction on $k$. When $k=0$, Lemma 2.15 is already proved in the previous section (with $b_{0}=1 / 2$ ). Let us now assume that inequalities (2.53) and (2.52) are true at the level $k-1$ (i.e. if we replace $k$ by $k-1$ ). We will show that they are true at the level $k$.

We will choose an (arbitrary) number $b_{k}>0$ such that $1>b_{k}>b_{k}^{2}>b_{k-1}, b_{0}$. (For example, one can put $b_{0}=1 / 2$ and then $b_{k}=\left(b_{k-1}\right)^{1 / 3}$ by recurrence.) What will be important for us is that $b_{0} / b_{k}, b_{k-1} / b_{k}^{2}$ and $b_{0} / b_{k}^{2}$ are positive numbers which are strictly smaller than 1 .

It follows from Lemmas 2.13 and 2.14 that there exist two positive numbers $c_{1}$ and $c_{2}$ (which do not depend on $n$ ) such that we have, for any $n \in \mathbb{N}$ :

$$
\left\|\psi_{n+1}\right\|_{k} \leqslant c_{1}\left(\left\|\psi_{n}\right\|_{0}\left\|\psi_{n}\right\|_{k}+\left\|\psi_{n}\right\|_{k-1}+\left\|\psi_{n}\right\|_{0}\left\|\phi_{n}\right\|_{k-1}+\left\|\psi_{n}\right\|_{0}^{2}\left\|\phi_{n}\right\|_{k}\right)
$$

and

$$
\left\|\phi_{n+1}\right\|_{k}-\left\|\phi_{n}\right\|_{k} \leqslant c_{2}\left(\left\|\psi_{n}\right\|_{k}+\left\|\psi_{n}\right\|_{0}\left\|\phi_{n}\right\|_{k}\right)
$$

We will now prove inequalities (2.53) and (2.52) by induction on $n$. There exists a natural number $n_{0}$ such that for any $n>0$ we have

$$
Q_{1}:=D_{0}\left(\frac{b_{0}}{b_{k}}\right)^{2^{n}}+\left(\frac{b_{k-1}}{b_{k}^{2}}\right)^{2^{n}}+D_{0}\left(\frac{b_{0}}{b_{k}^{2}}\right)^{2^{n}}+D_{0}^{2}\left(\frac{b_{0}}{b_{k}}\right)^{2^{n+1}} \leqslant \frac{1}{c_{1}}
$$

and

$$
Q_{2}:=\left(b_{k}\right)^{2^{n}}+D_{0}\left(b_{0}\right)^{2^{n}} \leqslant \frac{2^{-n-1}}{c_{2}}
$$

By choosing $D_{k}$ large enough, we can assume that inequalities (2.53) and (2.52) are satisfied for any $n \leqslant n_{0}$. We will also assume that $D_{k} \geqslant D_{k-1}$. Let us now show that if inequalities (2.53) and (2.52) are satisfied for some $n \geqslant n_{0}$ then they are still satisfied when we replace $n$ by $n+1$. (This is the last step in our induction process.)

Indeed, for $\left\|\psi_{n+1}\right\|_{k}$, using inequality (2.54) and the induction hypothesis, we get

$$
\begin{aligned}
& \left\|\psi_{n+1}\right\|_{k} \leqslant c_{1}\left(\left\|\psi_{n}\right\|_{k}\left\|\psi_{n}\right\|_{0}+\left\|\psi_{n}\right\|_{k-1}+\left\|\psi_{n}\right\|_{0}\left\|\phi_{n}\right\|_{k-1}+\left\|\psi_{n}\right\|_{0}^{2}\left\|\phi_{n}\right\|_{k}\right) \\
& \quad \leqslant c_{1}\left(D_{k}\left(b_{k}\right)^{2^{n}} D_{0}\left(b_{0}\right)^{2^{n}}+D_{k-1}\left(b_{k-1}\right)^{2^{n}}+D_{0}\left(b_{0}\right)^{2^{n}} D_{k-1}+D_{0}^{2}\left(b_{0}\right)^{2^{n+1}} D_{k}\right) \\
& \quad \leqslant D_{k} c_{1}\left(\left(b_{k}\right)^{2^{n}} D_{0}\left(b_{0}\right)^{2^{n}}+\left(b_{k-1}\right)^{2^{n}}+D_{0}\left(b_{0}\right)^{2^{n}}+D_{0}^{2}\left(b_{0}\right)^{2^{n+1}}\right) \\
& \quad=D_{k} c_{1} Q_{1}\left(b_{k}\right)^{2^{n+1}} \leqslant D_{k}\left(b_{k}\right)^{2^{n+1}} .
\end{aligned}
$$

Similarly, for $\left\|\phi_{n+1}\right\|_{k}$ we have:

$$
\begin{aligned}
& \left\|\phi_{n+1}\right\|_{k} \leqslant\left\|\phi_{n}\right\|_{k}+c_{2}\left(\left\|\psi_{n}\right\|_{k}+\left\|\psi_{n}\right\|_{0}\left\|\phi_{n}\right\|_{k}\right) \\
& \quad \leqslant D_{k}\left(1-2^{-n}\right)+c_{2}\left(D_{k}\left(b_{k}\right)^{2^{n}}+D_{0}\left(b_{0}\right)^{2^{n}} \cdot D_{k}\right) \\
& \quad \leqslant D_{k}\left(1-2^{-n}\right)+D_{k} c_{2}\left[\left(b_{k}\right)^{2^{n}}+D_{0}\left(b_{0}\right)^{2^{n}}\right] \\
& \quad=D_{k}\left(1-2^{-n}\right)+D_{k} c_{2} Q_{2} \leqslant D_{k}\left(1-2^{-n}\right)+D_{k} 2^{-n-1}=D_{k}\left(1-2^{-n-1}\right) .
\end{aligned}
$$

End of the proof of Theorem 2.3. - Inequality (2.53) is a sufficient condition for the $C^{k}$-smoothness of $\phi_{\infty}$ (provided that $k$ does not exceed the smoothness class of $\Gamma$ ), because 
it implies in particular that $\sum_{n=1}^{\infty}\left\|\Psi_{n}\right\|_{k} \preceq \sum_{n=1}^{\infty}\left\|\psi_{n}\right\|_{k}<\infty$, which in turns implies that the sequence of maps $\left(\phi_{n}\right)$ converges in the $C^{k}$-topology, by Lemma 2.11. Thus the homomorphism $\phi_{\infty}: \Gamma \rightarrow G$ has the same smoothness class as $\Gamma$.

Remark. - If we start with a near-homomorphism from $\Gamma$ to a compact Lie group $H$ different from $G$, then our iterative averaging method still yields a homomorphism from $\Gamma$ to $H$. So we get a generalization of the cited Grove-Karcher-Ruh's result [12] about approximation of nearhomomorphisms by homomorphisms.

\subsection{Proof of Theorem 2.5}

Recall that the linear part of the Poisson structure $\Pi$ at the fixed point $m$ is isomorphic to the Lie-Poisson structure on $\mathfrak{g}^{*}$. Theorem 2.3 allows us to linearize $\Gamma$ near $m$ without the symplectic structure. The corresponding linear action of $G=G_{m}$ must be (isomorphic to) the coadjoint action, so without losing generality we may assume that $P$ is a neighborhood of 0 in $\mathfrak{g}^{*}$, and the orbits on $P$ near 0 are nothing but the coadjoint orbits (though the symplectic form on each orbit may be different from the standard one). But then, as was shown by Ginzburg and Weinstein [11] using a standard Moser's path argument, since $G$ is compact, the Poisson structure on $P$ is actually locally isomorphic to the Lie-Poisson structure of $\mathfrak{g}^{*}$. We can now apply the following proposition to finish the proof of Theorem 2.5:

Proposition 2.16. - If $G$ is a (not necessarily connected) compact Lie group and $\mathfrak{g}$ is its Lie algebra, then any proper symplectic groupoid $(\Gamma, \omega) \rightrightarrows U$ with a fixed point 0 whose base Poisson manifold is a neighborhood $U$ of 0 in $\mathfrak{g}^{*}$ with the Lie-Poisson structure and whose isotropy group at 0 is $G$ is locally isomorphic to $T^{*} G \rightrightarrows \mathfrak{g}^{*}$.

Proof of Proposition 2.16. - Without loss of generality, we can assume that $\Gamma$ is source-locally trivial.

We will first prove the above proposition for the case when $G$ is connected. The Lie algebra $\mathfrak{g}$ can be written as a direct sum $\mathfrak{g}=\mathfrak{s} \oplus \mathfrak{l}$, where $\mathfrak{s}$ is semisimple and $\mathfrak{l}$ is Abelian. Denote by $\left(f_{1}, \ldots, f_{n}, h_{1}, \ldots, h_{m}\right)$ a basis of linear functions on $\mathfrak{g}^{*}$, where $f_{1}, \ldots, f_{n}$ correspond to $\mathfrak{s}$ and $h_{1}, \ldots, h_{m}$ correspond to $l$. Then the vector fields $X_{s^{*} f_{i}}, X_{s^{*} h_{j}}$ generate a Hamiltonian action of $\mathfrak{g}$ on $(\Gamma, \omega)$. When restricted to the isotropy group $G=G_{0}$ over the origin of $\mathfrak{g}^{*}$, the vector fields $X_{s^{*} f_{i}}, X_{s^{*} h_{j}}$ become left-invariant vector fields on $G$, and the action of $\mathfrak{g}$ integrates to the right action of $G$ on itself by multiplication on the right. Assume that the above Hamiltonian action of $\mathfrak{g}$ integrates to a right action of $G$ on $\Gamma$. Then we are done. Indeed, since the action is free on $G_{0}$, we may assume, by shrinking the base space $U$, that the action is free on $\Gamma$. Then one can verify directly that the map $(g, y) \mapsto \varepsilon\left(\operatorname{Ad}_{g}^{*} y\right) \circ g, g \in G, y \in U$, where $\varepsilon: U \rightarrow \Gamma$ denotes the identity section and $\circ g$ denotes the right action by $g$, is a symplectic isomorphism between the restriction of the standard symplectic groupoid $G \times \mathfrak{g}^{*} \cong T^{*} G \rightrightarrows \mathfrak{g}^{*}$ to $U \subset \mathfrak{g}^{*}$ and $\Gamma$.

In general, the action of $\mathfrak{g}$ on $\Gamma$ integrates to an action of the universal covering of $G$ on $\Gamma$, which does not factor to an action of $G$ on $\Gamma$ if the Abelian part of $G$ is nontrivial, i.e. $\mathfrak{l} \neq 0$. So we may have to change the generators of this $\mathfrak{g}$ action, by changing $h_{1}, \ldots, h_{m}$ to new functions $h_{i}^{\prime}$ which are still Casimir functions of $\mathfrak{g}^{*}$. Such a change of variables (leaving $f_{i}$ intact) will be a local Poisson isomorphism of $\mathfrak{g}^{*}$. We want to choose $h_{i}^{\prime}$ so that the Hamiltonian vector fields $X_{s^{*} h_{i}^{\prime}}$ are periodic, i.e. they generate Hamiltonian $\mathbb{T}^{1}$-actions.

Note that for each $y \in U \subset \mathfrak{g}^{*}$, the isotropy group $G_{y}=s^{-1}(y) \cap t^{-1}(y)$ of $\Gamma$ at $y$ admits a canonical injective homomorphism to $G$ (via a priori non-symplectic local linearization of $\Gamma$ using Theorem 2.3). Denote by $\mathbb{T}_{0}^{m}$ the Abelian torus of dimension $m$ in the center of $G$ (the Lie algebra of $\mathbb{T}_{0}^{m}$ is $\mathfrak{l}$ ). The coadjoint action of $\mathbb{T}_{0}^{m}$ on $\mathfrak{g}^{*}$ is trivial. It follows that each isotropy

$4^{\text {e }}$ SÉRIE - TOME $39-2006-\mathrm{N}^{\circ} 5$ 
group $G_{y}$ contains a torus $\mathbb{T}_{y}^{m}$ whose image under the canonical injection to $G$ is $\mathbb{T}_{0}^{m}$. For each $q \in \Gamma$, denote $\mathbb{T}_{q}^{m}=q \cdot \mathbb{T}_{s(q)}^{m}=\mathbb{T}_{t(q)}^{m} . q$. Note that if $r \in \mathbb{T}_{q}^{m}$ then $\mathbb{T}_{r}^{m}=\mathbb{T}_{q}^{m}$.

Choose a basis $\gamma_{1}, \ldots, \gamma_{m}$ of 1 -dimensional sub-tori $\mathbb{T}^{m}$. Translate them to each point $q \in \Gamma$ as above, we get $m$ curves $\gamma_{1, q}, \ldots, \gamma_{m, q} \subset \mathbb{T}_{q}^{m} \forall q \in \Gamma$. Recall that, due to the fact that $\mathbb{T}_{0}^{m}$ lies in the center of $G$, these curves are well-defined and depend continuously on $q$.

Since $G=s^{-1}(0)=t^{-1}(0)$ is a Lagrangian submanifold of $\Gamma$, the symplectic form $\omega$ of $\Gamma$ is exact (near $G$ ) and we can write $\omega=\mathrm{d} \alpha$. Define $m$ functions $H_{i}, i=1, \ldots, m$, on $\Gamma$ via the following integral formula, known as Arnold-Mineur formula for action functions of integrable Hamiltonian systems [21]:

$$
H_{i}(q)=\int_{\gamma_{i, q}} \alpha .
$$

Denote by $\Gamma_{\text {reg }}$ the "regular" part of $\Gamma$, i.e. the set of points $q \in \Gamma$ such that $t(q)$ is a regular point of the Poisson structure $\Pi$ in $P$. Then $\Gamma_{\text {reg }}$ admits a natural symplectically complete foliation by isotropic submanifolds $K_{q}=q \cdot G_{s(q)}$, and since $\Gamma$ is proper, these submanifolds are compact. So this foliation may be viewed as the foliation by invariant tori of a proper noncommutatively integrable Hamiltonian system. Since $\gamma_{i, q} \subset K_{q} \forall i$, it follows from the classical Arnold-Liouville-Mineur theorem on action-angle variables of integrable Hamiltonian systems that $H_{i}$ are action functions, i.e. the Hamiltonian vector fields $X_{H_{i}}$ are periodic (of period 1) and generate $\mathbb{T}^{1}$-actions, and they are tangent to the isotropic submanifolds $K_{q}, q \in \Gamma$. This fact is true in $\Gamma_{\text {reg, }}$, which is dense in $\Gamma$, so by continuity it is true in $\Gamma$.

By construction, the action functions $H_{i}$ are invariant on the leaves of the dual coisotropic foliation of the foliation by $K_{g}, g \in \Gamma_{\text {reg }}$, so they project to (independent) Casimir functions on $P$. In other words, we have $m$ independent Casimir functions $h_{1}^{\prime}, \ldots, h_{m}^{\prime}$ such that $s^{*} h_{i}^{\prime}=$ $t^{*} h_{i}^{\prime}=H_{i}$.

The infinitesimal action of $\mathfrak{g}$ on $\Gamma$ generated by Hamiltonian vector fields $X_{s^{*} f_{i}}, X_{s^{*} h_{j}^{\prime}}$, where the functions $f_{1}, \ldots, f_{n}$ are as before, now integrates into an action of $S \times \mathbb{T}^{m}$ on $\Gamma$, where $S$ is the connected simply-connected semisimple Lie group with Lie algebra $\mathfrak{s}$. The group $S \times \mathbb{T}^{m}$ is a finite covering of $G$, i.e. we have an exact sequence $0 \rightarrow \mathcal{G} \rightarrow S \times \mathbb{T}^{m} \rightarrow G \rightarrow 0$, where $\mathcal{G}$ is a finite group. Indeed, by construction, for every element $g \in \mathcal{G} \subset S \times \mathbb{T}^{m}$, the action $\phi(g)$ of $g$ on $\Gamma$ is identity on the isotropy group $G$, and its differential at the neutral element $e \in G \subset \Gamma$ is also the identity map of $T_{e} \Gamma$. Since a finite power of $\phi(g)$ is the identity map on $\Gamma$, it follows that $\phi(g)$ itself is the identity map. Hence the action of $\mathcal{G}$ on $\Gamma$ is trivial, and the action of $S \times \mathbb{T}^{m}$ on $\Gamma$ factors to a Hamiltonian action of $G$ on $\Gamma$. The proposition is proved for the case when $G$ is connected.

Consider now the case $G$ is disconnected. Denote by $G^{0}$ the connected component of $G$ which contains the neutral element, and by $\Gamma^{0}$ the corresponding connected component of $\Gamma$ (we assume that the base $U$ is connected and sufficiently small). Then $\Gamma_{0}$ is a proper symplectic groupoid over $U$ whose isotropy group at 0 is $G^{0}$. According to the above discussion, $\Gamma^{0}$ can be locally symplectically linearized, i.e. we may assume that $\Gamma^{0}$ is symplectically isomorphic to $\left(T^{*} G^{0} \rightrightarrows \mathfrak{g}^{*}\right)_{U}$ with the standard symplectic structure. Consider a map $\phi: \Gamma \rightarrow G$, whose restriction to the isotropy group $G=s^{-1}(0) \cap t^{-1}(0)$ is identity, and whose restriction to $\Gamma^{0}$ is given by the projection $T^{*} G^{0} \cong G^{0} \times \mathfrak{g}^{*} \rightarrow G^{0}$ after the above symplectic isomorphism from $\Gamma^{0}$ to $\left(G^{0} \times \mathfrak{g}^{*} \rightrightarrows \mathfrak{g}^{*}\right)_{U}$. We can arrange so that $\phi(p)=\phi\left(p^{-1}\right)^{-1}$ for any $p \in \Gamma$, and also $\phi(p) . \phi(q)=\phi(p . q)$ for any $p \in \Gamma^{0}, q \in \Gamma$. (This is possible because $\left.\phi\right|_{\Gamma_{0}}: \Gamma_{0} \rightarrow G^{0}$ is a homomorphism.) Then the averaging process used in the proof of Theorem 2.3 does not change the value of $\phi$ on $\Gamma$. By repeating the proof of Theorem 2.3, we get a homomorphism $\phi_{\infty}: \Gamma \rightarrow G$, which coincides with $\phi$ on $\Gamma^{0}$. 
Identifying $\Gamma$ with $G \times U$ via the isomorphism $p \mapsto\left(\phi_{\infty}(p), s(p)\right)$ as in the proof of Theorem 2.3, and then with $\left(T^{*} G \rightrightarrows \mathfrak{g}^{*}\right)_{U}$, we will assume that $\Gamma$, as a Lie groupoid, is nothing but the restriction $\left(T^{*} G \rightrightarrows \mathfrak{g}^{*}\right)_{U}$ of the standard symplectic groupoid $T^{*} G \rightrightarrows \mathfrak{g}^{*}$ to $U \subset \mathfrak{g}^{*}$, and the symplectic structure $\omega$ on $\left(T^{*} G\right)_{U} \cong G \times U$ coincides with the standard symplectic structure $\omega_{0}$ on the connected component $\left(T^{*} G^{0}\right)_{U} \cong G^{0} \times U$. For each $\theta \in G / G^{0}$, we will denote the corresponding connected component of $G$ by $G^{\theta}$ and the corresponding connected component of $\Gamma$ by $\Gamma^{\theta}$. We will use Moser's path method to find a groupoid isomorphism of $\Gamma$ which moves $\omega$ to $\omega_{0}$.

Let $f: U \rightarrow \mathbb{R}$ be a function on $U$. Then the Hamiltonian vector fields $X_{s^{*} f}^{\omega}$ and $X_{s^{*} f}^{\omega_{0}}$ of $s^{*} f$ with respect to $\omega$ and $\omega_{0}$ are both invariant under left translations in $\Gamma$, and since they coincide in $\Gamma^{0}$ they must coincide in $\Gamma$, because any element in $\Gamma$ can be left-translated from an element in $\Gamma^{0}$. So we have a common Hamiltonian vector field $X_{s^{*} f}$ for both $\omega$ and $\omega_{0}$. Similarly, we have a common Hamiltonian vector field $X_{t^{*} f}$ for both $\omega$ and $\omega_{0}$. It means that $i_{X}\left(\omega-\omega_{0}\right)=0$ for any $X \in T_{p} s^{-1}(s(p))+T_{p} t^{-1}(t(p))$, which implies that $\omega-\omega_{0}$ is a basic closed 2-form with respect to the coisotropic singular foliation whose leaves are connected components of the sets $s^{-1}\left(s\left(t^{-1}(t(p))\right), p \in \Gamma\right.$. In particular, for any connected component $\Gamma^{\theta}$ of $\Gamma$, where $\theta \in G / G^{0}$, there is a unique closed 2-form $\beta_{\theta}$ on $U$, which is basic with respect to the foliation by the orbits of the coadjoint action of $G^{0}$ on $U$, such that

$$
\omega-\omega_{0}=s^{*} \beta_{\theta} \quad \text { on } \Gamma^{\theta} .
$$

The coadjoint action of $G$ on $U$ induces an action $\rho$ of $G / G^{0}$ on the space of connected coadjoint orbits (orbits of $G^{0}$ ) on $U$ : if $\mathcal{O}$ is a connected coadjoint orbit on $U$, then $\rho(\theta)(\mathcal{O})$ is the orbit $\operatorname{Ad}_{G^{\theta}}^{*} \mathcal{O}$. Since $\Gamma$ is a symplectic groupoid with respect to both $\omega$ and $\omega_{0}$, the closed 2 -form $\omega-\omega_{0}$ is also compatible with the product map in $\Gamma$. By projecting this compatibility condition to $U$, we get the following equality:

$$
\beta_{\theta_{1} \theta_{2}}=\beta_{\theta_{2}}+\rho\left(\theta_{2}\right)^{*} \beta_{\theta_{1}} \quad \forall \theta_{1}, \theta_{2} \in G / G_{0} .
$$

Since the 2 -forms $\beta_{\theta}$ are closed on $U$ which are basic with respect to the foliation by connected coadjoint orbits (i.e. orbits of the coadjoint action of $G^{0}$ ), we can write

$$
\beta_{\theta}=\mathrm{d} \alpha_{\theta},
$$

where $\alpha_{\theta}$ are 1-forms on $U$ which are also basic with respect to the foliation by connected coadjoint orbits. Indeed, write $\beta_{\theta}=\mathrm{d} \hat{\alpha}_{\theta}$, then define $\alpha_{\theta}$ by the averaging formula

$$
\alpha_{\theta}=\int_{G^{0}}\left(\operatorname{Ad}_{g}^{*}\right)^{*} \hat{\alpha}_{\theta} \mathrm{d} \mu_{G^{0}},
$$

where $\mu_{G^{0}}$ is the Haar measure on $G^{0}$. Then $\alpha_{\theta}$ is invariant with respect to the coadjoint action of $G^{0}$, and $\mathrm{d} \alpha_{\theta}=\beta_{\theta}$. One verifies easily that $\alpha_{\theta}$ must automatically vanish on vector fields tangent to the coadjoint orbits, or otherwise $\beta_{\theta}$ would not be a basic 2 -form.

Moreover, by averaging $\alpha_{\theta}$ with respect to the action of $G / G^{0}$ via the formula

$$
\alpha_{\theta}^{\text {new }}=\frac{1}{\left|G / G^{0}\right|} \sum_{\theta^{\prime} \in G / G^{0}}\left(\alpha_{\theta^{\prime} \theta}-\rho(\theta)^{*} \alpha_{\theta}\right),
$$

$4^{\text {e }}$ SÉRIE - TOME $39-2006-\mathrm{N}^{\circ} 5$ 
we may assume that the 1 -forms $\alpha_{\theta}$ satisfy the equation

$$
\alpha_{\theta_{1} \theta_{2}}=\alpha_{\theta_{2}}+\rho\left(\theta_{2}\right)^{*} \alpha_{\theta_{1}} \quad \forall \theta_{1}, \theta_{2} \in G / G^{0} .
$$

Consider the vector field $Z$ on $\Gamma$ defined by

$$
s^{*} \alpha_{\theta}=i_{Z} \omega=i_{Z} \omega_{0} \quad \text { on } \Gamma_{\theta} .
$$

One verifies directly that the flow $\phi_{Z}^{t}$ of $Z$ preserves the groupoid structure of $\Gamma$, and $\phi_{Z}^{1}$ moves $\omega$ to $\omega_{0}$.

\section{Momentum maps}

In this section, to avoid dealing with quotient spaces which are orbifolds instead of manifolds (with boundary and corners), we will only consider groupoids whose isotropy groups are coadconnected (see Definition 2.7), even when we do not mention it explicitly.

\subsection{Affine structure on base spaces}

In this subsection we will show that the orbit space $X=P / \Gamma$ of a proper (quasi-)symplectic groupoid $(\Gamma \rightrightarrows P, \omega+\Omega$ ) whose isotropy groups are coad-connected is not only a manifold with locally polyhedral boundary (Corollary 2.8 ), but it also admits a natural (locally flat) affine structure which makes it into an integral affine manifold with locally convex polyhedral boundary (the boundary may be empty). It means that $X$ admits an atlas with charts modelled on convex subsets with non-empty interior in $\mathbb{R}^{k}$, and the transformations maps are integral affine, i.e. are given by elements of the integral affine group $G L(k, \mathbb{Z}) \ltimes \mathbb{R}^{k}$. Moreover, near every point $X$ is locally affine-equivalent to a Weyl chamber of a compact Lie group (points in the interior of $X$ correspond to tori while point on the boundary correspond to non-commutative compact Lie groups). We will assume that $X=P / \Gamma$ is connected.

Consider first the standard symplectic groupoid $T^{*} G \rightrightarrows \mathfrak{g}^{*}$ of a compact Lie group $G$. In this case, the orbit space $\mathfrak{g}^{*} / T^{*} G$ is the space of coadjoint orbits of $G$ on $\mathfrak{g}^{*}$ and can be identified naturally with a Weyl chamber $\mathfrak{t}_{+}^{*}$ (here $\mathfrak{t}$ denotes a Cartan subalgebra of $\mathfrak{g}$ ). The affine structure on $\mathfrak{g}^{*} / T^{*} G \cong \mathfrak{t}_{+}^{*}$ is induced from the standard affine structure on $t_{+}^{*}$. There is another equivalent definition of this affine structure on $\mathfrak{g}^{*} / T^{*} G$, which is more intrinsic and can be generalized to arbitrary proper (quasi-)symplectic groupoids. Let us do it immediately for a general proper symplectic groupoid $(\Gamma \rightrightarrows P, \omega)$.

Recall from Corollary 2.8 that the orbit space $P / \Gamma$ is a manifold with locally convex polyhedral boundary, and the dimension $k=\operatorname{dim} P / \Gamma$ is also the rank (i.e. the dimension of a Cartan torus) of each isotropic group of $\Gamma \rightrightarrows P$. Moreover, the points on the boundary of $P / \Gamma$ correspond to the points on $P$ whose isotropy groups are essentially non-Abelian, while the points in the interior of $P / \Gamma$ correspond to the points on $P$ whose isotropy groups are essentially Abelian (i.e. the connected component of identity is a torus of dimension $k$ ). For each point $z \in P$ which projects to an interior point of $P / \Gamma$, denote by $\mathbb{T}_{z}^{k}$ the connected component of its isotropy group $\Gamma_{z}$. Choose a basis $\left(\gamma_{1}^{z}, \ldots, \gamma_{k}^{z}\right)$ of $H_{1}\left(\mathbb{T}_{z}^{k}, \mathbb{Z}\right)$, and move it continuously when $z$ moves (via the Gauss-Manin connection). Note that $\mathbb{T}_{z}^{k}$ is an isotropic submanifold in $\Gamma$, and hence the symplectic form $\omega$ is exact in a neighborhood of $\mathbb{T}_{z}^{k}$. Denote by $\alpha$ a primitive of $\omega$, $\mathrm{d} \alpha=\omega$ in a neighborhood of $\mathbb{T}_{z}^{k}$ in $\Gamma$, and define the following functions (in a neighborhood 
of $z$ in $P$ ):

$$
F_{i}(z)=\int_{\gamma_{i}^{z}} \alpha, \quad i=1, \ldots, k .
$$

(This is the same as the well-known Mineur-Arnold formula for action functions of integrable Hamiltonian systems [21].) It is clear that these functions are independent. If we change $\alpha$ to another primitive of $\omega$, then $F_{i}$ are changed by additive constants, and the closed 1-forms $\mathrm{d} F_{i}$ are not changed.

It is easy to check that, due to the compatibility of $\omega$ with $\Gamma$, and more precisely to the fact that for any $g \in G_{z}, T_{g} G_{z}$ is symplectically orthogonal to $T_{g} \Gamma_{\mathcal{O}}$ where $\mathcal{O}$ denotes the orbit through $z$, these local closed 1 -forms $\mathrm{d} F_{i}$ vanish on the orbits of $\Gamma$ on $P$. If $z^{\prime}$ is another point lying on the orbit $\mathcal{O}(z)$ of $z$ (not necessarily on the same connected component of the orbit), then there is a unique natural way to transport the basis $\left(\gamma_{1}^{z}, \ldots, \gamma_{k}^{z}\right)$ of $H_{1}\left(\mathbb{T}_{z}^{k}, \mathbb{Z}\right)$ to a basis $\left(\gamma_{1}^{z^{\prime}}, \ldots, \gamma_{k}^{z^{\prime}}\right)$ of $H_{1}\left(\mathbb{T}_{z^{\prime}}^{k}, \mathbb{Z}\right)$ via the action of $\Gamma$. Again, by the compatibility of $\omega$ with $\Gamma$, this transportation moves closed 1-forms $\left(\mathrm{d} F_{1}, \ldots, \mathrm{d} F_{k}\right)$ near $z$ to closed 1-forms $\left(\mathrm{d} F_{1}, \ldots, \mathrm{d} F_{k}\right)$ near $z^{\prime}$ in a unique natural way. These facts mean that the closed 1 -forms $\left(\mathrm{d} F_{1}, \ldots, \mathrm{d} F_{k}\right)$ can be extended in a natural single-valued way to independent closed 1-forms on a neighborhood of $\mathcal{O}(z)$ on $P$ and then projected to independent closed 1 -forms on a neighborhood of $z / \Gamma$ in the orbit space $P / \Gamma$.

If we replace the basis $\left(\gamma_{1}^{z}, \ldots, \gamma_{k}^{z}\right)$ by another basis of $H_{1}\left(\mathbb{T}_{z}^{k}, \mathbb{Z}\right)$ (say by the holonomy obtained by moving $z$ along a loop in $P$ ), then the vector-valued closed 1 -form $\left(\mathrm{d} F_{1}, \ldots, \mathrm{d} F_{k}\right)$ is changed by a linear transformation given by an element of $G L(k, \mathbb{Z})$. It means that while the projection of $\mathrm{d} F_{1}, \ldots, \mathrm{d} F_{k}$ to $P / \Gamma$ is only locally defined on (the interior of) $P / \Gamma$ and depends on the choice of a basis of $H_{1}\left(\mathbb{T}_{z}^{k}, \mathbb{Z}\right)$, it determines in a unique way a natural integral affine structure on (the interior) of $P / \Gamma$ (integral means that the linear parts of the transformation maps lie in $G L(k, \mathbb{Z})$ ). To see that this affine structure extends well to the boundary of $P / \Gamma$, we simply go back to the local model $T^{*} G \rightrightarrows \mathfrak{g}^{*}$, invoking Theorem 2.5. In this local model, it is easy to check that the affine structure defined intrinsically above in the interior of $\mathfrak{g}^{*} / T^{*} G$ coincides with the affine structure obtained by identifying $\mathfrak{g}^{*} / T^{*} G$ with $t_{+}^{*}$.

Remark. - If $G_{1}$ is a connected finite covering of a connected compact Lie group $G$ then their corresponding groupoids $T^{*} G \rightrightarrows \mathfrak{g}^{*}$ and $T^{*} G_{1} \rightrightarrows \mathfrak{g}^{*}$ induce the same affine structure on $t_{+}^{*}$ but maybe different integral affine structures: the lattice of constant integral closed 1-forms on $t_{+}^{*}$ coming from $T^{*} G_{1} \rightrightarrows \mathfrak{g}^{*}$ is a sublattice of the one coming from $T^{*} G \rightrightarrows \mathfrak{g}^{*}$.

The situation is similar in the case of proper quasi-symplectic groupoids. A technical difference is that, since $\omega$ is not closed in general, we have to replace formula (3.1) by another formula in order to define the analogs of $\mathrm{d} F_{i}$ : Let $z:[0,1] \rightarrow P$ be a small path in $P$ which projects to the interior of $P / \Gamma$. Denote by $\left(\gamma_{1}^{r}, \ldots, \gamma_{k}^{r}\right)$ a basis of $H^{1}\left(\mathbb{T}_{z(r)}^{k}, \mathbb{Z}\right)$ which depends continuously on $r \in[0,1]$ (via the Gauss-Manin connection). Then define $\alpha_{i}$ to be a unique closed 1-form (defined in a neighborhood of $z(0)$ ) such that for any such small path $z$ we have

$$
\int_{z(0)}^{z(1)} \alpha_{i}=\int_{C} \omega
$$

where $C$ is a cylinder in $\bigcup_{r \in[0,1]} \mathbb{T}_{z(r)}^{k}$ whose intersection with each $\mathbb{T}_{z(r)}^{k}$ is a simple closed curve representing $\gamma_{i}^{r}$. The compatibility of $\omega$ with $\Gamma$ implies that the above 1 -form $\alpha_{i}$ is welldefined (i.e. does not depend on the choice of $C$ ), vanishes on the orbits of $\Gamma$ and is invariant

$4^{\mathrm{e}}$ SÉRIE - TOME $39-2006-\mathrm{N}^{\circ} 5$ 
under the action of $\Gamma$ in a natural sense, so that it can be projected to $P / \Gamma$. The condition $\mathrm{d} \omega=t^{*} \Omega-s^{*} \Omega$ implies that the isotropy groups in $\Gamma$ are tangent to the kernel of $\mathrm{d} \omega$, which in turn guaranties that this 1 -form $\alpha_{i}$ is closed. This is the replacement for the 1 -form $\mathrm{d} F_{i}$ of the symplectic case. The rest is absolutely similar to the symplectic case.

Recall [32] that two quasi-symplectic groupoids $\left(\Gamma^{1} \rightrightarrows P^{1}, \omega^{1}+\Omega^{1}\right)$ and $\left(\Gamma^{2} \rightrightarrows P^{2}, \omega^{2}+\Omega^{2}\right)$ are called Morita equivalent if there exists a quasi-Hamiltonian equivalence bimodule, i.e. a manifold $M$ with the following properties:

(i) $\Gamma^{1}$ acts on $M$ from the left with momentum map $\mu^{1}, \Gamma^{2}$ acts on $M$ from the right with momentum map $\mu^{2}$, and the two actions commute. Moreover, the actions of $\Gamma^{1}$ and $\Gamma^{2}$ on $M$ are free, the momentum maps are submersions, and the orbits of $\Gamma_{1}$ on $M$ are precisely the fibers of $\mu_{2}$ and vice versa.

(ii) There is a 2 -form $\sigma$ on $M$ which makes it into a quasi-Hamiltonian $\Gamma^{1} \times \overline{\Gamma^{2}}$-space, where $\overline{\Gamma^{2}}$ means $\left(\Gamma^{2} \rightrightarrows P^{2},-\omega-\Omega\right)$, and the (left) action of $\Gamma^{1} \times \overline{\Gamma^{2}}$ on $M$ is given by $\left(g_{1}, g_{2}\right) \cdot m:=g_{1} \cdot m \cdot g_{2}^{-1}$.

When two Lie groupoids are Morita equivalent, they have the same orbit spaces up to isomorphisms. In the case of proper quasi-symplectic groupoids, one can check easily that their orbit spaces also have the same integral affine structure, because we can "move" formula (3.2) from one groupoid to another via a quasi-Hamiltonian equivalence bimodule. Summarizing, we have:

THEOREM 3.1. - If $(\Gamma \rightrightarrows P, \omega+\Omega)$ is a proper quasi-symplectic groupoid with coadconnected isotropy groups, then its orbit space $P / \Gamma$ admits a natural structure of an integral affine manifold which near each point is locally affine-isomorphic to a Weyl chamber of a compact Lie group, and which depends only on the Morita equivalence class of $(\Gamma \rightrightarrows P, \omega+\Omega)$.

Remark. - The affine structure on $P / \Gamma$ can be lifted to $P$ to become a transverse affine structure to the orbits of $\Gamma$ in $P$. It can also be lifted to $\Gamma$ to become a transverse affine structure to the foliation in $\Gamma$ given by submanifolds $s^{-1}\left(t^{-1}(m)\right), m \in P$. Of course, one has a similar intrinsic definition for these transverse affine structures in $P$ and $\Gamma$.

Example 3.2. - Consider the AMM (Alekseev-Malkin-Meinrenken) groupoid [32]: it is the action groupoid $G \times G \rightrightarrows G$ of the conjugation action of a compact Lie group $G$, equipped with a natural quasi-symplectic structure arising from the theory of group-valued momentum maps [1]. Xu [32] showed a natural equivalence between quasi-Hamiltonian spaces with $G$-valued momentum maps and quasi-Hamiltonian spaces of the AMM groupoid. The orbit space of the AMM groupoid is naturally affine-equivalent to a Weyl alcove of $G$. In particular it is a convex affine polytope.

\subsection{Affinity and local convexity of momentum maps}

Consider a Hamiltonian action of a symplectic groupoid $(\Gamma \rightrightarrows P, \omega)$ on a symplectic manifold $(M, \sigma)$, i.e. an action of $\Gamma$ on $M$ which is compatible with the symplectic forms in the following sense (see [20]): the graph $\{(g, x, g . x) \mid g \in \Gamma, x \in P, s(g)=\mu(x)\}$ of the action is an isotropic submanifold in $(\Gamma, \omega) \times(M, \sigma) \times(M,-\sigma)$. Here $\mu$ denotes the momentum map of the action; it is a Poisson map from $M$ to $P$. This is a generalization of Hamiltonian actions of Lie groups, because, as was shown by Mikami and Weinstein [20], there is a natural 1-1 correspondence between Hamiltonian actions of a given Lie group $G$ with equivariant momentum maps and Hamiltonian actions of the symplectic groupoid $T^{*} G \rightrightarrows \mathfrak{g}^{*}$, in the following sense: If $T^{*} G \rightrightarrows \mathfrak{g}^{*}$ acts on a symplectic manifold $(M, \sigma)$ with momentum map $\mu$, then $\mu$ is also the equivariant 
momentum map of a Hamiltonian action of $G$ on $M$ defined as follows:

$$
g \cdot x=\left(L_{g} \mu(x)\right) \cdot x,
$$

where $g \in G, x \in M, L_{g}$ means left translation by $g$ in $T^{*} G, g . x$ means the action of $g \in G$ on $x$ and $\left(L_{g} \mu(x)\right) . x$ means the action of $L_{g} \mu(x) \in T^{*} G$ on $x$ (note that $s\left(L_{g} \mu(x)\right)=\mu(x)$ ). Conversely, if $\mu$ is the momentum map of a Hamiltonian action of $G$ on $(M, \sigma)$, then formula (3.3) defines a Hamiltonian action of $T^{*} G \rightrightarrows \mathfrak{g}^{*}$ on $(M, \sigma)$ with the same momentum map. In particular, the orbits of the action of $G$ on $(M, \sigma)$ are the same as the orbits of the action of $T^{*} G \rightrightarrows \mathfrak{g}^{*}$. Remark that $G$ can be disconnected, in which case, by a Hamiltonian $G$-action we mean a symplectic action of $G$ on a symplectic manifold $(M, \sigma)$ together with a $G$-equivariant Poisson map $\mu: M \rightarrow \mathfrak{g}^{*}$ (the equivariant momentum map).

Theorem 2.5, together with the above equivalence between Hamiltonian $G$-actions and Hamiltonian ( $\left.T^{*} G \rightrightarrows \mathfrak{g}^{*}\right)$-actions, leads immediately to the following proposition:

Proposition 3.3. - Denote by $\mu$ the momentum map of a Hamiltonian action of a proper symplectic groupoid $(\Gamma \rightrightarrows P, \omega)$ on a symplectic manifold $(M, \sigma)$. Let $m$ be an arbitrary point of $M$, and denote by $N$ a submanifold in $P$ which intersects the symplectic leaf $\mathcal{O}(\mu(m))$ of $\mu(m)$ in $P$ transversally at $\mu(m)$. Then there is a small neighborhood $B$ of $\mu(m)$ in $N$ with the following properties:

(i) $M_{B}:=\mu^{-1}(B)$ is a symplectic submanifold of $M$ which intersects the orbits of the action of $\Gamma$ on $M$ transversally.

(ii) $\left(\Gamma_{B} \rightrightarrows B, \omega\right)$ is isomorphic to $\left(T^{*} G \rightrightarrows \mathfrak{g}^{*}\right)_{U}$, where $G=G_{\mu(m)}$ is the isotropy group of $\mu(m)$ and $U$ is a neighborhood of 0 in $\mathfrak{g}^{*}$. Denote by $\phi: B \rightarrow \mathfrak{g}^{*}$ a corresponding isomorphism from $B$ to $U \subset \mathfrak{g}^{*}$.

(iii) The induced Hamiltonian action of $\left(\Gamma_{B} \rightrightarrows B, \omega\right)$ on $\left(M_{B}, \sigma\right)$ is equivalent to a Hamiltonian action of $G$ on $\left(M_{B}, \sigma\right)$ with the equivariant momentum map $\phi \circ \mu: M_{B} \rightarrow \mathfrak{g}^{*}$.

Proof. - The proof is straightforward.

More generally, we may consider a quasi-Hamiltonian space $(M, \sigma)$, in the sense of Xu [32], of a proper quasi-symplectic groupoid $(\Gamma \rightrightarrows P, \omega+\Omega)$. It means that $\Gamma$ acts on $M$, and the following compatibility and weak nondegeneracy conditions are satisfied:

(i) $\mathrm{d} \sigma=\mu^{*} \Omega$, where $\mu$ denotes the momentum map.

(ii) The graph of the action is isotropic with respect to the 2 -form $\omega \oplus \sigma \oplus(-\sigma)$.

(iii) $\forall m \in M, \operatorname{ker} \sigma_{m}=a_{*}\left(T_{\mu(m)} s^{-1}(\mu(m)) \cap \operatorname{ker} \omega_{\mu(m)}\right)$. Here $a$ denotes the action map $s^{-1}(\mu(m)) \rightarrow M, a(g):=g . m$. In particular, if $\omega$ is nondegenerate then $\sigma$ is also nondegenerate.

Similarly to the case of Hamiltonian actions of proper symplectic groupoids, Corollary 2.6 leads to the following proposition, whose proof is straightforward:

PROPOSITION 3.4. - Denote by $\mu$ the momentum map of a quasi-Hamiltonian space $(M, \sigma)$ of a proper quasi-symplectic groupoid $(\Gamma \rightrightarrows P, \omega+\Omega)$. Let $m$ be an arbitrary point of $M$, and denote by $N$ a submanifold in $P$ which intersects the symplectic leaf $\mathcal{O}(\mu(m))$ of $\mu(m)$ in $P$ transversally at $\mu(m)$. Then there is a neighborhood $B$ of $\mu(m)$ in $N$ and a primitive 2-form $\beta$ of the pull-back of $\Omega$ to $B\left(\mathrm{~d} \beta=\Omega_{B}\right)$, with the following properties:

(i) $\left(M_{B}:=\mu^{-1}(B), \sigma-\mu^{*} \beta\right)$ is a symplectic submanifold of $M$ which intersects the orbits of the action of $\Gamma$ on $M$ transversally.

(ii) $\left(\Gamma_{B} \rightrightarrows B, \omega+s^{*} \beta-t^{*} \beta\right)$ is a proper symplectic groupoid which is isomorphic to $\left(T^{*} G \rightrightarrows \mathfrak{g}^{*}\right)_{U}$, where $G=G_{\mu(m)}$ is the isotropy group of $\mu(m)$ and $U$ is a neighborhood of 0 in $\mathfrak{g}^{*}$. Denote by $\phi: B \rightarrow \mathfrak{g}^{*}$ a corresponding isomorphism from $B$ to $U \subset \mathfrak{g}^{*}$.

$4^{\mathrm{e}}$ SÉRIE - TOME $39-2006-\mathrm{N}^{\circ} 5$ 
(iii) The induced action of $\left(\Gamma_{B} \rightrightarrows B, \omega+s^{*} \beta-t^{*} \beta\right)$ on $\left(M_{B}, \sigma-\mu^{*} \beta\right)$ is Hamiltonian, and is equivalent to the Hamiltonian action of $G$ on $\left(M_{B}, \sigma-\mu^{*} \beta\right)$ associated to the momentum map $\phi \circ \mu: M_{B} \rightarrow \mathfrak{g}^{*}$.

The above proposition means that locally, near a level set of the momentum map and after going to a slice, a quasi-Hamiltonian space of a proper quasi-symplectic groupoid is the same as a Hamiltonian space of a compact Lie group. So it is natural to expect that many results concerning momentum maps of Hamiltonian actions of compact Lie groups apply to quasiHamiltonian spaces of proper quasi-symplectic groupoids as well. We will be interested in their local convexity properties, so let us recall the following local convexity result in the "classical" setting:

Consider a Hamiltonian action of a coad-connected compact Lie group $G$ on a symplectic manifold $(M, \sigma)$ with an equivariant momentum map $\mu: M \rightarrow \mathfrak{g}^{*}$. We can factorize $\mu$ by the action of $G$ to get a kind of reduced momentum map:

$$
\mu_{/ G}: M / G \rightarrow \mathfrak{g}^{*} / G \cong \mathfrak{t}_{+}^{*} .
$$

We will assume that $\mu_{/ G}^{-1}(0)=\mu^{-1}(0) / G$ is not empty, and denote by $N / G$ a connected component of $\mu_{/ G}^{-1}(0)$. (The subset $N$ of $M$ is not necessarily connected, but $N / G$ is connected; we assume that $M$ itself is without boundary.)

PROPOSITION 3.5 (Kirwan [16]). - With the above notations, there is a neighborhood $U / G$ of $N / G$ in $M / G$ such that $\mu_{/ G}(U / G)$ is a neighborhood of 0 in a closed convex polyhedral cone $C$ of vertex 0 in $\mathbf{t}_{+}^{*}$, and that for any $c \in \mu_{/ G}(U / G)$ the reduced level set $\mu_{/ G}^{-1}(c) \cap U / G$ is connected.

Proof. - The above proposition was proved by Kirwan [16] using Morse theory. See also, e.g., $[15,27,17]$ for additional information about local properties of momentum maps. Strictly speaking, these papers consider only the case when $G$ is connected, but the case when $G$ is disconnected but coad-connected is the same because of $G$-equivariance.

Consider now a quasi-Hamiltonian space $(M, \sigma)$ of a proper quasi-symplectic groupoid $(\Gamma \rightrightarrows P, \omega+\Omega)$ with momentum map $\mu$. Factorize $\mu$ by the action of $\Gamma$ to get the reduced momentum map

$$
\mu_{/ \Gamma}: M / \Gamma \rightarrow P / \Gamma
$$

The orbit space $M / \Gamma$ of the action of $\Gamma$ on $M$ is foliated by the connected components of the preimages of the reduced map $\mu_{/ \Gamma}$. Denote by $\widehat{M}$ the quotient space of this (singular) foliation (together with the induced topology). Then the reduced momentum map $\mu_{/ \Gamma}$ projects to a map

$$
\widehat{\mu}: \widehat{M} \rightarrow P / \Gamma .
$$

We will call $\widehat{\mu}$ the transverse momentum map. We will show that $\widehat{M}$ and $\widehat{\mu}$ enjoy very good affine properties:

THEOREM 3.6. - Let $(M, \sigma)$ be a quasi-Hamiltonian space of a proper quasi-symplectic groupoid $(\Gamma \rightrightarrows P, \omega+\Omega$ ) with coad-connected isotropy groups, with momentum map $\mu$. Then with the above notations we have:

(i) $\widehat{M}$ is a manifold with locally convex polyhedral boundary (the boundary can be empty).

(ii) There is a natural affine structure of $\widehat{M}$ which makes it into an affine manifold which near boundary points is locally affine-isomorphic to convex polyhedral cones. 
(iii) The transverse momentum map $\widehat{\mu}$ is locally injective and affine (i.e. the pull-back of an affine function is affine).

Proof. - The affine structure on $\widehat{M}$ can be defined either by the pull-back via $\widehat{\mu}$ of the affine structure on $P / \Gamma$ once we establish that $\widehat{\mu}$ is locally injective with locally polyhedral image, or by local 1 -forms defined by a formula similar to formula (3.2): replace $\omega$ by $\sigma$, and 1-cycles on $\mathbb{T}_{z}^{k}(z \in P)$ by 1 -cycles on $\mathbb{T}_{m}^{k}=\mathbb{T}_{z}^{k} \cdot m(m \in M$ with $\mu(m)=z)$. The two definitions are equivalent, and the obtained affine structure is actually an integral affine structure. Since the statements of the above theorem are local, we can work locally, and replace $(\Gamma \rightrightarrows P, \omega+\Omega)$ by a standard symplectic groupoid $T^{*} G \rightrightarrows \mathfrak{g}^{*}$, in view of Theorem 2.5 and Corollary 2.6 and a result of $\mathrm{Xu}$ [32] which says that Morita equivalent quasi-symplectic groupoids have equivalent quasi-Hamiltonian spaces, i.e. we are reduced to the case of a Hamiltonian action of a compact Lie group on a symplectic manifold. But then it becomes nothing more than Proposition 3.5. Details are left to the reader.

Remark. - One can describe the boundary points of $P / \Gamma$ and of $\widehat{M}$ via the degeneration of 1 -cycles on tori. For example, when a point $z$ in the interior of $P / \Gamma$ goes to a boundary point, then the torus $\mathbb{T}_{z}^{k}$ becomes a non-commutative compact group, and (at least) one of the 1-cycles on $\mathbb{T}_{z}^{k}$ vanishes homotopically, and the local affine function corresponding to that cycle admits a minimum or maximum value on the corresponding boundary face of $P / \Gamma$. For a point $m$ of $M$ which goes to a boundary point of $\widehat{M}$ after the projection $M \rightarrow \widehat{M}$ (we assume that $M$ is without boundary), the situation is similar: the torus $\mathbb{T}_{m}^{k}$ collapses to a smaller torus, either because the torus $\mathbb{T}_{z}^{k}$ (with $s(z)=\mu(m)$ ) degenerates to a non-commutative group (i.e. we get a boundary point of $\widehat{M}$ which maps to a boundary point of $P / \Gamma$ ), or the action of $\mathbb{T}_{z}^{k}$ degenerates (i.e. there is a subtorus whose action becomes trivial), or both.

Remark. - In the case of Hamiltonian torus actions, the fact that $\widehat{M}$ is an affine manifold with locally convex polyhedral boundary was probably first pointed out in [4].

\subsection{Global convexity of momentum maps}

Theorem 3.6 allows us to reduce the problem of convexity of momentum maps to a problem concerning affine maps between locally convex affine manifolds . For example, when the orbit space $P / \Gamma$ can be affinely embedded or at least immersed into $\mathbb{R}^{k}$ (it happens if $P / \Gamma$ is simplyconnected), we can use the following two simple lemmas about affine maps to prove global convexity:

LEMMA 3.7. - Let $X$ be a connected compact locally convex affine manifold (with boundary), and $\phi: X \rightarrow \mathbb{R}^{k}$ a locally injective affine map from $X$ to $\mathbb{R}^{k}$ with the standard affine structure. Then $\phi$ is injective, and its image $\phi(X)$ is convex in $\mathbb{R}^{k}$.

Proof. - The proof is elementary. Take an arbitrary point $x \in X$. For each non-trivial vector $v \in T_{x} X$ (if $x$ lies on the boundary of $X$ then $T_{x} X$ means the convex tangent cone of $X$ at $x$ ), denote by $l_{v}$ the maximal affine segment lying in $X$ which begins at $x$ and going in the direction of $v$. Because $\phi$ is affine locally injective, the map $\left.\phi\right|_{l_{v}}: l_{v} \rightarrow \phi\left(l_{v}\right)$ is affine injective, and $\phi\left(l_{v}\right)$ is an affine segment in $\mathbb{R}^{k}$. Because $X$ is compact, $\phi(X)$ is compact and therefore $\phi\left(l_{v}\right)$ and $l_{v}$ must be compact too, i.e. $l_{v}$ is a closed bounded segment. Denote by $y_{v}$ the other end of $l_{v}$. Then $y_{v}$ lies on the boundary $\partial X$ of $X$. It follows easily from the local convexity of the boundary of $X$ that the union $\bigcup_{v \in T_{x} X} l_{v}$ is an open subset of $X$. Note that $\bigcup_{v \in T_{x} X} l_{v}$ is also closed in $X$. Indeed, if $y_{n} \in l_{v_{n}}, \lim y_{n}=y \neq x$, then we can assume (after taking a subsequence of $\left(y_{n}\right)$ and resizing $\left.\left(v_{n}\right)\right)$ that $0 \neq \lim v_{n}=v \in T_{x} M$, and it follows easily from the compactness that

$4^{\mathrm{e}}$ SÉRIE - TOME $39-2006-\mathrm{N}^{\circ} 5$ 
$y \in l_{v}$. Since $X$ is closed, we have $X=\bigcup_{v \in T_{x} X} l_{v}$, i.e. $X$ is star-shaped with respect to $x$. It follows from the local injectivity of $\phi$ at $x$ that $\phi$ is in fact injective, and $\phi(X)$ is star-shaped with respect to $\phi(x)$. But since $x$ is arbitrary, it means that $\phi(X)$ is convex.

LEMMA 3.8. - Let $X$ be a connected locally convex affine manifold, and $\phi: X \rightarrow \mathbb{R}^{k}$ a proper locally injective affine map from $X$ to $\mathbb{R}^{k}$ with the standard affine structure. Then $\phi$ is injective, and its image $\phi(X)$ is convex in $\mathbb{R}^{k}$.

Proof. - Absolutely similar to the proof of Lemma 3.7, via the fact that $X=\bigcup_{v \in T_{x} X} l_{v}$. Another proof goes as follows. Denote by $B_{n}$ the closed ball of radius $n$ centered at $\phi(x)$ in $\mathbb{R}^{k}$, and by $X_{n}$ the connected component of $\phi^{-1}\left(B_{n}\right)$ which contains $x$. Then one checks easily that $X_{n} \subset X_{n+1}, \bigcup_{n=1}^{\infty} X_{n}=X$, and each $X_{n}$ is compact locally convex. Hence, by Lemma 3.7, the restriction of $\phi$ to $X_{n}$ is injective and $\phi\left(X_{n}\right)$ is convex. Since $\phi\left(X_{n}\right) \subset \phi\left(X_{n+1}\right)$, it follows that $\phi(X)=\bigcup_{n=1}^{\infty} \phi\left(X_{n}\right)$ is convex.

Remark. - The above lemmas are along the lines of the "local-global principle" of convexity $[4,15]$; here we make this principle simpler by formulating it in terms of pure affine geometry.

Many known convexity theorems of momentum maps concern the case when $P / \Gamma$ is contractible, and can be recovered from the above two lemmas and Theorem 3.6. These include, for example:

- Kirwan's convexity theorem [16], which was conjectured and partially proved by Guillemin and Sternberg [13]: If a compact Lie group $G$ acts in a Hamiltonian way on a connected compact symplectic manifold $M$ with an equivariant momentum map $\mu: M \rightarrow \mathfrak{g}^{*}$, and $t_{+}^{*}$ denotes a Weyl chamber in $\mathfrak{g}^{*}$, then $\mu(M) \cap \mathfrak{t}_{+}^{*}$ is a convex polytope. In this case $P / \Gamma \cong \mathfrak{t}_{+}^{*}$, and Kirwan's theorem follows from Theorem 3.6 and Lemma 3.7. The generalization of Kirwan's theorem to the case of noncompact symplectic manifolds with proper momentum maps [15] follows from Theorem 3.6 and Lemma 3.8.

- Flaschka-Ratiu's convexity theorem for momentum maps of Poisson actions of compact Poisson-Lie groups [9]: again $P / \Gamma \cong t_{+}^{*}$.

- Alekseev-Malkin-Meinrenken's convexity theorem for group-valued momentum maps [1]: $P / \Gamma$ is a Weyl alcove.

- Weinstein's convexity theorem for certain Hamiltonian actions of a noncompact semisimple Lie group $G$ which admits a compact Cartan subgroup [30, Theorem 3.3]: here $P / \Gamma$ may be identified with a Weyl chamber of a compact Cartan subalgebra. A special case of this theorem with $G=S p(2 k, \mathbb{R})$ is a beautiful result concerning frequencies of positive-definite quadratic Hamiltonian functions [30, Theorem 4.1] reproduced below (Theorem 3.9). The paper [30] is actually one of the original motivations for the study of proper groupoids and momentum maps suggested to us by Weinstein.

THEOREM 3.9 (Weinstein [30]). - For any positive-definite quadratic Hamiltonian function $H$ on the standard symplectic space $\mathbb{R}^{2 k}$, denote by $\phi(H)$ the $k$-tuple $\lambda_{1} \leqslant \cdots \leqslant \lambda_{k}$ of frequencies of $H$ ordered nondecreasingly, i.e. $H$ can be written as $H=\sum \lambda_{i}\left(x_{i}^{2}+y_{i}^{2}\right) / 2$ in a canonical coordinate system. Then for any two given positive nondecreasing $n$-tuples $\lambda=\left(\lambda_{1}, \ldots, \lambda_{k}\right)$ and $\gamma=\left(\gamma_{1}, \ldots, \gamma_{k}\right)$, the set

$$
\Phi_{\lambda, \gamma}=\left\{\phi\left(H_{1}+H_{2}\right) \mid \phi\left(H_{1}\right)=\lambda, \phi\left(H_{2}\right)=\gamma\right\}
$$

is a closed, convex, locally polyhedral subset of $\mathbb{R}^{k}$.

Remark. - The above set $\Phi_{\lambda, \gamma}$ is closed but not bounded. For example, when $k=1$ then $\Phi_{\lambda, \gamma}$ is a half-line. 
There are cases when $P / \Gamma$ cannot be immersed into $\mathbb{R}^{k}$. An example is the situation of locally Hamiltonian torus actions studied by Giacobbe [10], where $P / \Gamma$ is isomorphic the quotient of $\mathbb{R}^{k}$ by a lattice. Then the image $\mu(M) / \Gamma$ of a proper momentum map $\mu$ in $P / \Gamma$ may or may not be locally convex, due to overlapping. For example, one can easily construct a convex polytope in $\mathbb{R}^{k}$ such that its image under the projection from $\mathbb{R}^{k}$ to $\mathbb{T}^{k}=\mathbb{R}^{k} / \mathbb{Z}^{k}$ is not locally convex. But one can still talk about global convexity after taking the universal covering of $P / \Gamma$.

Let us formulate some global convexity theorems based on the above discussions. First consider the case when $P / \Gamma$ is simply-connected. Then $P / \Gamma$ can be immersed into $\mathbb{R}^{k}$ (where $n$ is the dimension of $P / \Gamma$ ) by an integral affine map: a local integral affine system of coordinate on $P / \Gamma$ can be extended globally because there is no monodromy. Denote by $\mathfrak{j}: P / \Gamma \rightarrow \mathbb{R}^{k}$ such an immersion (it is unique up to integral affine automorphisms of $\mathbb{R}^{k}$ ). Then the map

$$
\mathfrak{j} \circ \widehat{\mu}: \widehat{M} \rightarrow \mathbb{R}^{k},
$$

where $\mu$ is the momentum map of a (quasi-)Hamiltonian $\Gamma \rightrightarrows P$ manifold $(M, \sigma)$, is a locally injective integral affine map. When $M$ is compact then $\widehat{M}$ is also compact, and Lemma 3.7 applies. When $M$ is noncompact, things are more subtle: the composed map $j \circ \widehat{\mu}$ may be nonproper even when $\mu$ is proper, so we need an additional condition besides the properness of $\mu$. For example, that $\mathfrak{j}$ is an embedding and its image $\mathfrak{j}(P / \Gamma)$ closed in $\mathbb{R}^{k}$ (then $\mathfrak{j} \circ \widehat{\mu}$ will be proper provided that $\mu$ is proper, and we can apply Lemma 3.8). Or that $\mathrm{j}$ is an embedding and $\mathrm{j}(P / \Gamma)$ is convex (not necessarily closed) in $\mathbb{R}^{k}$ : then we can write $\mathrm{j}(P / \Gamma)=\bigcup_{k=n}^{\infty} K_{n}$ where $K_{n} \subset K_{n+1}$ and each $K_{n}$ is a compact convex subset of $\mathbb{R}^{k}$, and we can use arguments absolutely similar to the above proof of Lemma 3.8 to show that the image of $j \circ \widehat{\mu}$ is convex if $\mu$ is proper. Thus we have:

THEOREM 3.10. - Let $(M, \sigma)$ be a connected quasi-Hamiltonian manifold of a proper quasisymplectic groupoid $(\Gamma \rightrightarrows P, \omega+\Omega)$ with coad-connected isotropy groups, with a proper momentum map $\mu$. Assume that the orbit space $P / \Gamma$ of $\Gamma$ is simply-connected, and denote by $\mathfrak{j}: P / \Gamma \rightarrow \mathbb{R}^{k}$ an integral affine immersion from $P / \Gamma$ to $\mathbb{R}^{k}$. Assume that at least one of the following additional conditions is satisfied:

(1) $M$ is compact.

(2) $\mathfrak{j}$ is an embedding and $\mathfrak{j}(P / \Gamma)$ is closed in $\mathbb{R}^{k}$.

(3) $\mathfrak{j}$ is an embedding and $\mathfrak{j}(P / \Gamma)$ is convex in $\mathbb{R}^{k}$.

Then the transverse momentum map $\widehat{\mu}$ and the composed map $\mathrm{j} \circ \widehat{\mu}$ are injective, and the image $\mathfrak{j} \circ \widehat{\mu}(\widehat{M})=\mathfrak{j}(\mu(M) / \Gamma)$ is a convex subset in $\mathbb{R}^{k}$ with locally polyhedral boundary. (We do not count boundary points which lie in the closure of $\mathrm{j}(\mu(M) / \Gamma)$ but not in $\mathfrak{j}(\mu(M) / \Gamma)$.) In particular, $\widehat{M}$ with its integral affine structure is isomorphic to a convex subset of $\mathbb{R}^{k}$ with locally polyhedral boundary.

Remark. - In the above theorem, one may say that $(M, \sigma)$ itself is a convex (quasi-)Hamiltonian manifold, in analogy with Knop [17]. The space $\widehat{M}$ with its affine structure is not only intrinsically locally convex, but it is also intrinsically globally convex, and the convexity of $\mathfrak{j}(\mu(M) / \Gamma)$ is just a manifestation of this intrinsic convexity.

Consider now the case when $P / \Gamma$ cannot be affinely immersed into $\mathbb{R}^{k}$ (in particular it is not simply-connected). Then its universal covering is a simply-connected affine manifold and hence can be immersed into $\mathbb{R}^{k}$. In fact, there is a minimal connected covering of $P / \Gamma$ which can be immersed into $\mathbb{R}^{k}$ : it corresponds to the subgroup of the fundamental group of $P / \Gamma$ which is precisely the isotropy group of the monodromy representation of the flat affine structure of $P / \Gamma$. We will denote this minimal "flattening" covering by $\widetilde{P / \Gamma}$, and again by $\mathrm{j}: \widetilde{P / \Gamma} \rightarrow \mathbb{R}^{k}$ the

$4^{\text {e }}$ SÉRIE - TOME $39-2006-\mathrm{N}^{\circ} 5$ 
corresponding integral affine immersion. The locally injective affine map $\widehat{\mu}: \widehat{M} \rightarrow P / \Gamma$ can be lifted to a locally injective affine map

$$
\widetilde{\widehat{\mu}}: \widetilde{\widehat{M}} \rightarrow \widetilde{P / \Gamma}
$$

where $\widetilde{\widehat{M}}$ denotes the (connected) covering of $\widehat{M}$ corresponding to the subgroup of the fundamental group of $\widehat{M}$ consisting of those elements which are mapped by $\widehat{\mu}$ into the isotropy group of the monodromy representation of the affine structure of $P / \Gamma$. It follows from the definition that $\widetilde{\widehat{\mu}}$ has the following property: if $x$ and $y$ are two points in $\widetilde{\widehat{M}}, x \neq y$, which project to a same point on $\widehat{M}$, then $\widetilde{\widehat{\mu}}(x) \neq \widetilde{\widehat{\mu}}(y)$. This property implies that $\widetilde{\widehat{\mu}}$ is a proper map, provided that $\mu$ (or $\widehat{\mu}$ ) is proper. Note that $\widetilde{\widehat{M}}$ is not compact in general, even when $M$ is compact. We have the following analog of Theorem 3.10:

THEOREM 3.11. - Let $(M, \sigma)$ be a connected quasi-Hamiltonian manifold of a proper quasi-symplectic groupoid $(\Gamma \rightrightarrows P, \omega+\Omega)$ with coad-connected isotropy groups, with a proper momentum map $\mu$. With the above notations, assume that at least one of the following conditions is satisfied:

(1) $\widetilde{\widehat{M}}$ is compact.

(2) $\mathrm{j}: \widetilde{P / \Gamma} \rightarrow \mathbb{R}^{k}$ is injective and its image is convex.

(3) $\mathrm{j}: \widetilde{P / \Gamma} \rightarrow \mathbb{R}^{k}$ is injective and its image is closed.

Then $\mathrm{j} \circ \widetilde{\widehat{\mu}}: \widetilde{\widehat{M}} \rightarrow \mathbb{R}^{k}$ is an injective integral affine map and its image is a convex subset of $\mathbb{R}^{k}$ with locally polyhedral boundary. (We do not count boundary points which lie in the closure of the image but not in the image itself.)

Remark. - In this paper we did not touch the "real" case, i.e. the case with an anti-symplectic involution studied by Duistermaat [8] and other people. We would conjecture that the main results of this paper have analogs in the case with an (anti-symplectic) involution.

\section{Acknowledgements}

The paper arises from numerous discussions with Alan Weinstein and aims to study some of his questions and carry out some of his ideas on proper groupoids and convexity. The main results of the paper were first announced at Alan Day, a colloquium in honor of Alan Weinstein held at Utrecht University in March 2003. The paper is dedicated to him. I am also thankful to Ping Xu for telling me about quasi-symplectic groupoids and their connections with different momentum map theories, and to Tudor Ratiu, Eugene Lerman, and Reyer Sjamaar for interesting discussions on convexity and connectedness properties of momentum maps. Thanks are due to Philippe Monnier for finding out some errors in a previous version of the paper. I would also like to thank the referees for numerous suggestions which helped me improve the paper.

\section{REFERENCES}

[1] Alekseev A., Malkin A., Meinrenken E., Lie group valued moment maps, J. Differential Geom. 48 (3) (1998) 445-495.

[2] Bochner S., Compact groups of differentiable transformations, Ann. of Math. (2) 46 (1945) 372-381.

[3] Bursztyn H., Crainic M., Weinstein A., Zhu C., Integration of twisted Dirac brackets, Duke Math. J. 123 (3) (2004) 549-607. 
[4] Condevaux M., Dazord P., Molino P., Géométrie du moment, in: Séminaire Sud-Rhodanien I, Publications du Département de Mathématiques, Univ. Claude Bernard, Lyon I, 1988, pp. 131-160.

[5] Coste A., Dazord P., Weinstein A., Groupoïdes symplectiques, in: Publications du Département de Mathématiques. Nouvelle Série A, vol. 2, in: Publ. Dép. Math. Nouvelle Sér. A, vol. 87, Univ. Claude Bernard, Lyon, 1987, pp. i-ii, pp. 1-62.

[6] Crainic M., Fernandes R.L., Integrability of Lie brackets, Ann. of Math. (2) 157 (2) (2003) 575 620.

[7] Dufour J.-P., Zung N.T., Poisson Structures and Their Normal Forms, Progr. Math., vol. 242, Birkhäuser, Basel, 2005.

[8] Duistermant J.J., Convexity and tightness for restrictions of Hamiltonian functions to fixed point sets of an antisymplectic involution, Trans. Am. Math. Soc. 275 (1983) 417-429.

[9] Flaschka H., Ratiu T., A convexity theorem for Poisson actions of compact Lie groups, Ann. Sci. École Norm. Sup. (4) 29 (6) (1996) 787-809.

[10] GiacobBe A., Convexity and multivalued Hamiltonians, Russ. Math. Surv. 55 (3) (2000) 578-580.

[11] Ginzburg V.L., Weinstein A., Lie-Poisson structure on some Poisson Lie groups, J. Amer. Math. Soc. 5 (2) (1992) 445-453.

[12] Grove K., Karcher H., Ruh E.A., Group actions and curvature, Invent. Math. 23 (1974) 31-48.

[13] Guillemin V., Sternberg S., Convexity properties of the moment mapping, Invent. Math. 67 (1982) 491-513.

[14] Haefliger A., Groupoïdes d'holonomie et classifiants, Astérisque 116 (1984) 70-97.

[15] Hilgert J., NeEB K.-H., Plank W., Symplectic convexity theorems and coadjoint orbits, Compos. Math. 94 (2) (1994) 129-180.

[16] KIRWAN F., Convexity properties of the moment mapping. III, Invent. Math. 77 (3) (1984) 547-552.

[17] KNOP F., Convexity of Hamiltonian manifolds, J. Lie Theory 12 (2) (2002) 571-582.

[18] Koszul J.-L., Sur certains groupes de transformations de Lie, in: Géométrie différentielle. Colloques internationaux du CNRS, Strasbourg, 1953, CNRS, Paris, 1953, pp. 137-141.

[19] LU J.-H., Momentum mappings and reduction of Poisson actions, in: Symplectic Geometry, Groupoids, and Integrable Systems (Berkeley, CA, 1989), in: Math. Sci. Res. Inst. Publ., vol. 20, Springer, New York, 1991, pp. 209-226.

[20] Mikami K., Weinstein A., Moments and reduction for symplectic groupoids, Publ. Res. Inst. Math. Sci. 24 (1) (1988) 121-140.

[21] Mineur H., Sur les systèmes mécaniques dans lesquels figurent des paramètres fonctions du temps. Étude des systèmes admettant $n$ intégrales premières uniformes en involution. Extension à ces systèmes des conditions de quantification de Bohr-Sommerfeld, Journal de l'École Polytechnique, Série III, 143ème année (1937) 173-191, 237-270.

[22] MoerdiJK I., Pronk D.A., Orbifolds, sheaves and groupoids, K-Theory 12 (1) (1997) 3-21.

[23] Moerdijk I., Mrcun J., Introduction to Foliations and Lie Groupoids, Cambridge Studies in Advanced Mathematics, vol. 91, Cambridge University Press, Cambridge, 2003, ix+173 pp.

[24] Molino P., Orbit-like foliations, in: Geometric Study of Foliations (Tokyo, 1993), World Scientific, River Edge, NJ, 1994, pp. 97-119.

[25] Monnier Ph., Zung N.T., Levi decomposition for smooth Poisson structures, J. Differential Geom. 68 (2) (2004) 347-395.

[26] Palais R.S., On the existence of slices for actions of non-compact Lie groups, Ann. of Math. (2) 73 (1961) 295-323.

[27] SJamaAR R., Convexity properties of the moment mapping re-examined, Adv. Math. 138 (1) (1998) 46-91.

[28] Weinstein A., Almost invariant submanifolds for compact group actions, J. Eur. Math. Soc. (JEMS) 2 (1) (2000) 53-86.

[29] WeInSTEIn A., Linearization problems for Lie algebroids and Lie groupoids, Lett. Math. Phys. 52 (1) (2000) 93-102.

[30] Weinstein A., Poisson geometry of discrete series orbits, and momentum convexity for noncompact group actions, Lett. Math. Phys. 56 (1) (2001) 17-30.

[31] Weinstein A., Linearization of regular proper groupoids, J. Inst. Math. Jussieu 1 (3) (2002) 493-511.

$4^{\mathrm{e}}$ SÉRIE - TOME $39-2006-\mathrm{N}^{\circ} 5$ 
[32] XU P., Momentum maps and Morita equivalence, J. Diff. Geom. 67 (2) (2004) 289-333.

[33] ZUnG N.T., Levi decomposition of analytic Poisson structures and Lie algebroids, Topology 42 (6) (2003) 1403-1420.

(Manuscrit reçu le 30 janvier 2006; accepté, après révision, le 5 septembre 2006.)

\section{Nguyen Tien ZUNG}

Laboratoire Émile Picard,

UFR MIG,

UMR 5580 CNRS,

Université Toulouse III,

118 route de Narbonne,

31062 Toulouse cedex, France

E-mail: tienzung@picard.ups-tlse.fr 